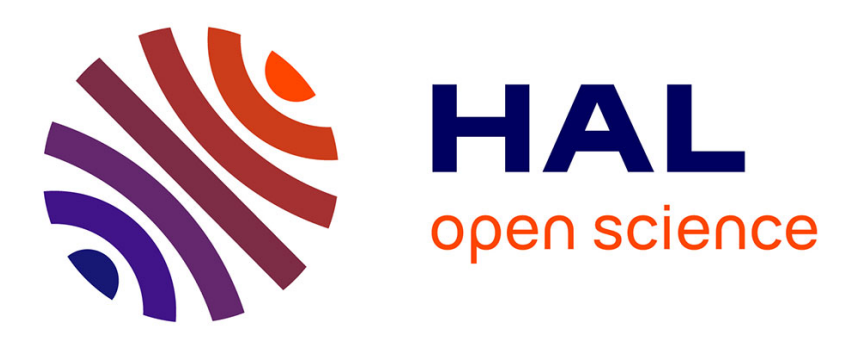

\title{
Cultivation of algae in photobioreactors for biodiesel production
}

Jeremy Pruvost

\section{To cite this version:}

Jeremy Pruvost. Cultivation of algae in photobioreactors for biodiesel production. Ashok Pandey; Christian Larroche; Claude-Gilles Dussap; Edgard Gnansounou; Samir Kumar Khanal; Steven Ricke. Biofuels: Alternative Feedstocks and Conversion Processes for the Production of Liquid and Gaseous Biofuels (Second Edition), Elsevier, pp.629-659, 2019, Biomass, Biofuels, Biochemicals, 978-0-12816856-1. 10.1016/B978-0-12-816856-1.00026-9 . hal-02539898

\section{HAL Id: hal-02539898 https://hal.science/hal-02539898}

Submitted on 20 Apr 2020

HAL is a multi-disciplinary open access archive for the deposit and dissemination of scientific research documents, whether they are published or not. The documents may come from teaching and research institutions in France or abroad, or from public or private research centers.
L'archive ouverte pluridisciplinaire HAL, est destinée au dépôt et à la diffusion de documents scientifiques de niveau recherche, publiés ou non, émanant des établissements d'enseignement et de recherche français ou étrangers, des laboratoires publics ou privés. 


\section{Cultivation of Algae in Photobioreactors for Biodiesel Production

\author{
Jeremy Pruvost \\ GEPEA, University of Nantes, CNRS, UMR6144, Saint-Nazaire, France
}

INTRODUCTION

Photosynthetic microorganisms such as microalgae and cyanobacteria (named for convenience "microalgae" in what follows, except when cited) have a high potential in biofuel production. Their main advantages are: solar production with higher surface productivities than plants, simultaneous consumption of inorganic carbon, allowing a null carbon balance exploitation, and possible production in closed systems, offering several advantages including an intensified, controlled production with very low environmental impact (no fertilizer is released and water can be reused). The high biodiversity of microalgae means that a variety of energy-rich substances can be produced, such as hydrogen by water photolysis, lipids for biodiesel, or biokerosene production, and sugars for biomass fermentation (methane) or gasification [1-8]. However, using microalgae for biofuels introduces several constraints, in particular, the need to set up mass-scale, cost-effective, and sustainable plant. This last constraint implies, for example, achieving a positive energy balance, which is not straightforward considering the different steps required to obtain usable biofuel (production, harvesting, and downstream processing of biomass into biofuel). Mass-scale production of microalgae has proved feasible for several decades, but in domains other than biofuel production [9]. Significant research and development efforts are still needed to define an integrated, efficient rproduction system meeting the specific constraints of the energy market.

This chapter is devoted to a key step in using algae for energy production purposes, namely the biomass production system. A specific technology is required. Microalgae cultivation possesses features common to bioreactors in general, such as thermal regulation, 
nutrient feeding procedures, $\mathrm{pH}$ regulation, and mixing for heat and mass transfer enhancement. However, a light supply is necessary for photosynthetic growth, with several consequences, in particular, the need for a dedicated cultivation system emphasizing large illuminated areas. Unlike other more classical bioprocesses where mixing tanks display standard geometries, cultivation systems for microalgae are characterized by a broad diversity, ranging from open ponds (open systems) to photobioreactor technology (closed systems). A detailed description of existing geometries can be found in the literature [10-13]. This chapter will present only a brief overview. Photobioreactor technology will be highlighted as it offers several advantages of special interest to biofuel production. However, as is wellknown, it also leads to more complex and costly processes, and is difficult to scale up for mass production on large land areas. Engineering breakthroughs are thus still needed before suitable systems can be set up. Recent scientific work has brought new insights into how such systems might be achieved, especially by clarifying the parameters governing photobioreactor productivities and establishing engineering bases to optimize and intensify them. These aspects will be presented here in the specific context of solar production. The case of production of oil-enriched microalgae for biodiesel application will also be considered.

\subsection{BASIC CONCEPTS OF PHOTOBIOREACTOR ENGINEERING}

\subsubsection{General Description}

Photosynthetic growth in standard autotrophic conditions is based on the assimilation, under illumination of inorganic carbon and mineral nutrients dissolved in the medium. The cultivation of photosynthetic microorganisms will thus require:

- A light supply (solar or artificial source, with an appropriate light spectrum in the photosynthetic active radiation (PAR) range, usually $0.4-0.7 \mu \mathrm{m}$ ),

- An inorganic carbon source (such as dissolved $\mathrm{CO}_{2}$ ),

- Mineral nutrients (major nutrients such as N, S, P sources and micronutrients as $\mathrm{Mg}, \mathrm{Ca}$, $\mathrm{Mn}, \mathrm{Cu}, \mathrm{Fe}$, etc.),

- Set culture conditions ( $\mathrm{pH}$, temperature).

Growth medium composition depends on the species cultivated. For a given species, mineral requirements can be ascertained using various methods, for example, direct measurement of their consumption, or elemental composition analysis. This is easy for major nutrients (a detailed explanation can be found in Pruvost et al. [14]), but can be very difficult for micronutrients, which may require specific analytical methods (see Cogne et al. [15]). Mineral requirements can be expressed in the form of a stoichiometric equation that can be used to prevent mineral limitation by adapting nutrient concentration as a function of biomass concentration achieved in the cultivation system [16]. Below are two examples for the fresh water species Chlamydomonas reinhardtii (Eq. 26.1) and Neochloris oleoabundans (Eq. 26.2):

$$
\begin{aligned}
& \mathrm{CO}_{2}+0.593 \mathrm{H}_{2} \mathrm{O}+0.176 \mathrm{NH}_{4}^{+}+0.007 \mathrm{SO}_{4}^{2-}+0.018 \mathrm{PO}_{4}^{3-} \rightarrow \\
& \mathrm{CH}_{1.781} \mathrm{O}_{0.437} \mathrm{~N}_{0.176} \mathrm{~S}_{0.007} \mathrm{P}_{0.018}+0.108 \mathrm{H}^{+}+1.127 \mathrm{O}_{2}
\end{aligned}
$$




$$
\begin{gathered}
\mathrm{CO}_{2}+0.751 \mathrm{H}_{2} \mathrm{O}+0.148 \mathrm{NO}_{3}{ }^{-}+0.014 \mathrm{SO}_{4}{ }^{2-}+0.012 \mathrm{PO}_{4}{ }^{3-}+0.212 \mathrm{H}^{+} \rightarrow \\
\mathrm{CH}_{1.715} \mathrm{O}_{0.427} \mathrm{~N}_{0.148} \mathrm{~S}_{0.014} \mathrm{P}_{0.012}+1.437 \mathrm{O}_{2}
\end{gathered}
$$

As for any biological production, a stoichiometric equation is useful for converting biomass growth rates into substrate or product rates, for example, to determine nutrient requirements (especially in terms of nitrogen, phosphorus, and sulfur sources for photosynthetic microorganisms). Eqs. (26.1) and (26.2) show, for example, the high biological requirement for $\mathrm{CO}_{2}$, opening perspectives for $\mathrm{CO}_{2}$ biological valorization by microalgae [17].

As an acid, $\mathrm{CO}_{2}$ has a direct influence on $\mathrm{pH}$. Its uptake leads to a progressive but significant basification of the medium [18]. Eqs. (26.1) and (26.2) also emphasize the difference due to the nitrogen source (ammonium for Chlamydomonas reinhardtii vs. nitrate for Neochloris oleoabundans). Oxygen release, for example, differs by $25 \%-30 \%$. In addition, ammonium consumption tends to lower the $\mathrm{pH}\left(\mathrm{H}^{+}\right.$release), while nitrate consumption tends to raise it $\left(\mathrm{H}^{+}\right.$ consumption). Special attention must therefore be paid to the nitrogen source when $\mathrm{pH}$ regulation is applied. In any case, $\mathrm{pH}$ will be affected by growth, with a significant influence of the carbon uptake due to its high consumption during photosynthetic growth. $\mathrm{pH}$ regulation will then be necessary to maintain an optimal value during cultivation (especially in the case of high volumetric productivities involving high nutrient consumption).

Most of the problems described previously (design of the medium composition, influence of biological uptake on physical, and chemical characteristics of the medium) are common to all classical bioprocesses. Light energy supply, however, is highly specific. Unlike dissolved nutrients, which can be assumed to be homogeneous in well-mixed conditions, light energy is heterogeneously distributed in the culture due to absorption and scattering by cells, independently of the mixing conditions (Fig. 26.1). As light is the principal energy source of photosynthesis, this simple fact makes microalgae cultivation systems different from other classical bioprocesses: specific approaches are thus needed for the design, optimization, and control of the cultivation system. Photosynthetic activity $(P)$ is directly related to the light received. This is usually represented as the light response curve as given in Fig. 26.1. This curve is characterized by a progressive saturation of photosynthesis with fluence rate $G$ up to a fluence rate of saturation $G_{s}$. For higher fluence rates, photoinhibition phenomena can occur with a negative influence on growth [19]. We also note that a threshold value of fluence rate is needed to obtain positive growth. This value is termed fluence rate of compensation $G_{C}$ (corresponding to the "compensation point of photosynthesis"). In cultivation systems, this nonlinear, complex response of photosynthesis has to be considered in combination with the light attenuation conditions. In extreme cases of high light illumination and high light attenuation (high biomass concentration), cells in different physiological states will cooccur: some may be photoinhibited (close to the light source) and some will receive no light (deep in the culture). Ideally, the control of the system would require taking all these processes into account, a far from trivial task.

\subsubsection{Characterization of the Incident PFD}

The light energy received by the cultivation system is represented by the hemispherical incident light flux density $q$, or photon flux density (PFD) as it is commonly termed in microalgae studies. For any light source, the PFD has to be expressed in the range of 


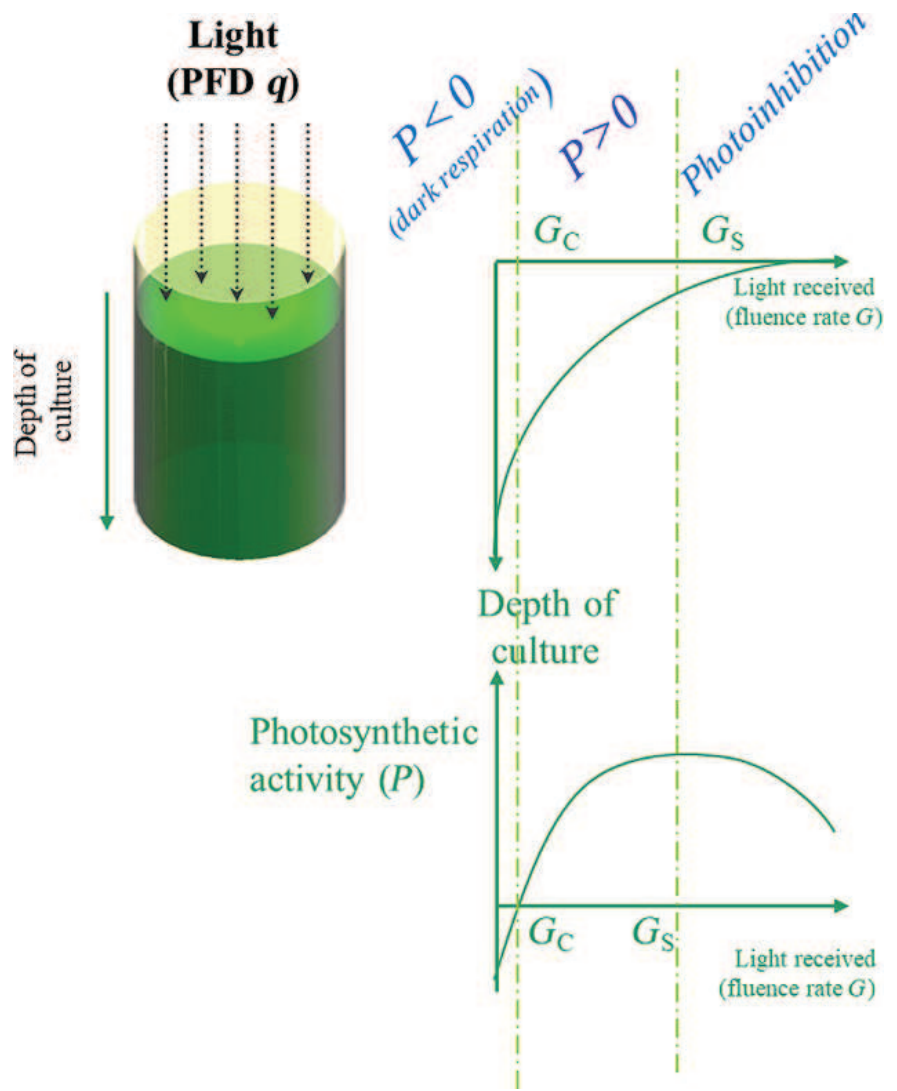

FIG. 26.1 Relation between light attenuation and photosynthetic growth in microalgal cultivation systems.

photosynthetic active radiation (PAR), in most cases in the $0.4-0.7 \mu \mathrm{m}$ bandwidth. For example, the whole solar spectrum at ground level covers the range $0.26-3 \mu \mathrm{m}$. The PAR range thus corresponds to almost $43 \%$ of the full solar energy spectrum.

As light is converted inside the culture volume, it is also necessary to add to PFD determination a rigorous treatment of radiative transfer inside the culture. This enables us, for example, to couple the resulting field of fluence rate with photosynthetic conversion of the algal suspension to simulate light-limited growth. However, this determination requires certain information. In addition to the PFD value, light source positioning with respect to the optical transparent surface of the cultivation system is important, as light penetration inside a turbid medium is affected by the incident polar angle $\theta$ of the radiation on the illuminated surface (Fig. 26.2). Ideally, beam and diffuse components of radiation should be considered separately. By definition, the direction of a beam of radiation, which represents direct radiation received from the light source, will define the incident polar angle $\theta$ with the illuminated surface. By contrast, diffuse radiation cannot be defined by a single incident angle but has an angular distribution over the illuminated surface (on a $2 \pi$ solid angle for a plane). We note that isotropic angular distribution is usually assumed, although an anisotropic distribution should ideally be considered because of the dependency of radiative transfer inside the culture volume on the angular nature of incident diffuse PFD. Both the incident angle and the degree of collimation of the light flux can be difficult to characterize. However, in most 


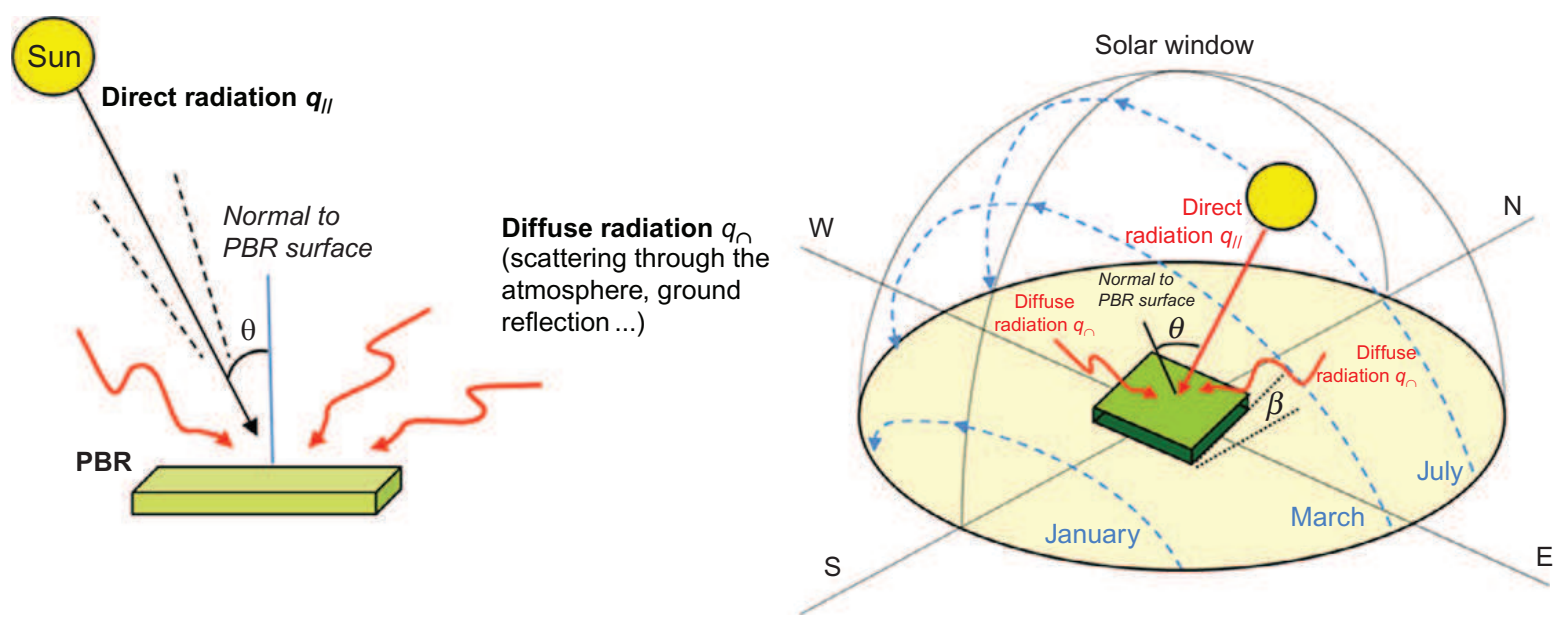

FIG. 26.2 Solar radiation on a microalgal cultivation system: incident angle and diffuse-beam radiations (left), evolution of solar sky path during the year (right).

artificial light cultivation systems, normal incidence is usually chosen as the most effective way to transfer light into the culture volume (less reflection on optical surfaces and better light penetration in the culture bulk). The PFD can also, in most cases, be assumed to be quasi-collimated (so we can consider the PFD as beam radiation only). However, these characteristics cannot be assumed in solar technology. The sun's displacement makes the incident angle time-dependent and so nonnormal incidence conditions will be encountered. Sunlight can also present a large proportion of diffuse radiation due to scattering through the atmosphere or by reflection from various surfaces, such as the ground. A detailed description of the respective consequences of neglecting incidence angle and direct/diffuse distribution effects in solar cultivation systems was recently published [20]. It was shown that each assumption led to an overestimation of $10 \%-20 \%$ in biomass productivity. When the two assumptions were combined (the simplest case of radiative transfer representation), an overestimation of up to $50 \%$ was obtained, emphasizing the relevance of an accurate consideration of the incident angle and direct/diffuse distribution in the radiative transfer modeling when applied to the solar case.

The PFD can be measured using a cosine quantum sensor (LI-190-SA, LI-COR, Lincoln, NE) with multi-point measurements to obtain an average over the illuminated surface [21-23]. The accuracy will closely depend on the average procedure, especially if the PFD is unevenly distributed. Actinometry could also be used for accurate characterization, as this is sensitive to all photons absorbed in the reaction volume. A detailed example of the experimental procedure in artificial light can be found in Pottier et al. [23]. In the case of sunlight, measurement is obviously also possible, but mathematical relations are also available to determine radiation conditions on a collecting surface as a function of the Earth's location, year period, and surface geometry [24]. An example was recently given by Sierra et al. [25] for a solar photobioreactor. Some commercial software packages integrating solar models are also available (METEONORM 6.0 software, www.meteonorm.com). These allow easy determination of irradiation conditions on a given surface. Such an approach is thus of particular interest in the case of solar production and was applied by author in several works [20, 26, 27]. 


\subsubsection{Light Attenuation in the Culture Bulk}

Owing to absorption and scattering by cells, light distribution in microalgae cultures is highly heterogeneous. This light distribution directly influences the light received by cells (termed the fluence rate $G$ ) and thus process efficiency. Light attenuation in a given cultivation system geometry depends on the optical properties and concentration of cells. Optical properties can be determined either experimentally or theoretically [23, 28, 29].

For a given culture, the field of fluence rate can be obtained experimentally using an underwater spherical sensor (US-SQS/A, Heinz Walz, LI-COR, Effeltrich, Germany). Such a quantum sensor measures the light from all incoming directions ( $4 \pi$ solid angle) in the PAR and has a small diameter $(3 \mathrm{~mm})$ allowing the fluence rate to be measured [23]. However, as any modification in cell concentration will modify light attenuation, this is of little interest. Radiative transfer models are to be preferred, of which further details are given in the next section.

\subsection{MODELING OF MICROALGAE CULTIVATION SYSTEMS}

\subsubsection{Mass Balance}

The mass balance relates concentration in the cultivation system to kinetic rates of biological production (biomass, $\mathrm{O}_{2}$ ) or consumption (nutrients, $\mathrm{CO}_{2}$ ) and system input and output. For a continuous system assuming perfectly mixed conditions, the biomass concentration $C_{x}$ is then given by [30-32]

$$
\frac{\mathrm{d} C_{x}}{\mathrm{~d} t}=\left\langle r_{x}\right\rangle-\frac{C_{x}}{\tau}=\left\langle r_{x}\right\rangle-D C_{x}
$$

with $C_{x}$ the biomass concentration, $\left\langle r_{x}\right\rangle$ the mean biomass volumetric growth rate in the system, and $\tau$ the residence time resulting from the liquid flow rate of the feed (fresh medium) (with $\tau=1 / D$, where $D$ is the dilution rate).

\subsubsection{Kinetic Modeling of Photosynthetic Growth}

Solving Eq. (26.3) involves determining the mean volumetric growth rate $\left\langle r_{x}\right\rangle$. This rate is linked to all possible limitations that can occur in the cultivation system. As will be shown later, light-limited conditions allow the best productivity to be obtained and they will be retained here as an example. With appropriate kinetic relations, other limitations can be considered (growth limitation by inorganic carbon or mineral nutrient concentration, temperature influence, etc.). The interested reader can refer to Fouchard et al. [33], where both light and nutrient limitations were modeled in the particular case of sulfur deprivation, which leads to starch accumulation and hydrogen production by Chlamydomonas reinhardtii.

There are numerous kinetic models linking photosynthetic microorganism growth to the light received $[34,35]$. For example, the following equations were applied for the cyanobacterium Arthrospira platensis [36] (Eq. 26.4) and the microalga Neochloris oleoabundans [32] (Eq. 26.5), respectively:

$$
r_{x}=\rho \bar{\varphi} \mathcal{A}=\rho_{\mathrm{M}} \frac{K}{K+G} \bar{\varphi} E_{\mathrm{a}} G C_{x}
$$




$$
r_{x}=\rho \bar{\varphi} \mathcal{A}-\mu_{\mathrm{s}} \mathcal{C}_{x}=\rho_{\mathrm{M}} \frac{K}{K+G} \bar{\varphi} E_{\mathrm{a}} G C_{x}-\mu_{\mathrm{s}} \mathcal{C}_{x}
$$

where $G$ is the fluence rate, $\rho_{M}$ the maximum energy yield for photon conversion, $\bar{\phi}$ the mass quantum yield for the $Z$-scheme of photosynthesis, $K$ the half saturation constant for photosynthesis, $E_{\mathrm{a}}$ the mass absorption coefficient, and $\mu_{\mathrm{s}}$ a specific respiration rate.

Both equations link the photosynthetic growth rate to the local specific rate of photons absorption and so to the local value of fluence rate $G$ inside the culture bulk $\left(A=E_{\mathrm{a}} G\right)$. As a prokaryotic cell, with therefore, a common electron carrier chain for photosynthesis and respiration, Arthrospira platensis displays negligible respiration in light [37]. This is not the case for microalgae, growth in light being the result of the biomass increase caused by photosynthesis in chloroplasts (anabolism) and its partial degradation by respiration in mitochondria (catabolism). It is thus necessary to introduce a catabolism respiration term, expressed here as a function of a constant specific respiration rate $\mu_{\mathrm{s}}$. This formulation is certainly oversimplified, as chloroplast and mitochondrial activities are not independent [38]. It was, however, shown to be sufficient in the case of Neochloris oleoabundans and could be retained in a first assumption at least for algae presenting a low respiration activity in light. For more information, the interested reader can refer to literature [39].

\subsubsection{Radiative Transfer Modeling}

Light attenuation conditions can be represented using radiative transfer models. Several examples can be found in the literature [40-44]. These models introduce assumptions in the radiative transfer equation, the solution of which requires complex numerical tools and long calculation times. However, several cultivation systems come under the so-called one-dimensional hypothesis, where light attenuation occurs mainly along a single direction perpendicular to the illuminated surface, termed the depth of culture $z$ (like a rectangular photobioreactor illuminated on one or both sides, cylindrical or spherical geometry with radial illumination). In this case, simple radiative models can be applied with relative accuracy. The simplest one is the Lambert-Beer law, but because of the scattering generated by cells, its use for microalgae is not recommended, especially when working in full light attenuation conditions [23, 35, 45-47]. The two-flux model offers a useful compromise, often giving a sufficiently accurate prediction of the radiation field in the context of photosynthetic microorganism cultivation $[23,36,40,48]$ with analytical solutions that facilitate further coupling with kinetic growth models. If geometries do not allow the one-dimensional hypothesis to be applied, numerical approaches will be required, entailing a significant computational effort [28].

An example of an analytical solution for the determination of the field of fluence rate is given below using the two-flux model. This example is given here for the solar case taking into account nonnormal incidence (thus introducing the incident angle $\theta$ ) with a separate treatment of the direct and diffuse parts of the radiation [26]. The PFD $q$ is thus divided into $q / /$ and $q_{\cap}\left(q=q_{/ /}+q_{\cap}\right)$,the direct and diffuse parts of the PFD respectively. The solution can be easily adapted for collimated radiation (diffuse radiation is then null) and normal incidence $(\theta=0)$. They are expressed here in Cartesian coordinates. For other geometries such as cylindrical ones, solutions can be adapted from works of Loubiere et al. [49] and Takache et al. [50]. 
We also note that with an increase in the computational effort, the field of fluence rate can be solved spectrally, taking into account the spectral distributions of PFD and of optical properties of photosynthetic microorganisms. This has already been applied for artificial light (see again Pottier et al. [23] and Farges et al. [51]).

The field of fluence rate for collimated radiation is given by.

$$
\frac{G_{\mathrm{col}}}{q_{/ /}}=\frac{2}{\cos \theta} \frac{(1+\alpha) \exp \left[-\delta_{\mathrm{col}}(z-L)\right]-(1-\alpha) \exp \left[\delta_{\mathrm{col}}(z-L)\right]}{(1+\alpha)^{2} \exp \left[\delta_{\mathrm{col}} L\right]-(1-\alpha)^{2} \exp \left[-\delta_{\mathrm{col}} L\right]}
$$

with $\delta_{\mathrm{col}}=\frac{\alpha C_{X}}{\cos \theta}\left(E_{\mathrm{a}}+2 b E_{\mathrm{s}}\right)$ the two-flux collimated extinction coefficient and $\alpha=\sqrt{\frac{E_{\mathrm{a}}}{\left(E_{\mathrm{a}} 2 b E_{\mathrm{s}}\right)}}$ the linear scattering modulus. $E_{\mathrm{a}}$ and $E_{\mathrm{s}}$ are, respectively, the mean (spectrally averaged over the PAR) mass absorption and scattering coefficients for the cultivated photosynthetic microorganism, $b$ the backward scattering fraction, and $C_{x}$ the biomass concentration in the culture medium.

For diffuse radiation, the following equation is obtained.

$$
\frac{G_{\mathrm{dif}}}{q_{\cap}}=4 \frac{(1+\alpha) \exp \left[-\delta_{\mathrm{dif}}(z-L)\right]-(1-\alpha) \exp \left[\delta_{\mathrm{dif}}(z-L)\right]}{(1+\alpha)^{2} \exp \left[\delta_{\mathrm{dif}} L\right]-(1-\alpha)^{2} \exp \left[-\delta_{\mathrm{dif}} L\right]}
$$

with $\delta_{\text {dif }}=2 \alpha C_{x}\left(E_{\mathrm{a}}+2 b E_{\mathrm{s}}\right)$ the two-flux diffuse extinction coefficient.

The total fluence rate (representing the amount of light impinging on algae) is finally given by simply summing the collimated and diffuse components.

$$
G(z)=G_{\text {col }}(z)+G_{\text {dif }}(z)
$$

Eqs. (26.6) and (26.7) show that penetrations of collimated and diffuse radiation inside the culture volume are markedly different. This will be especially important in solar conditions where the diffuse component of the radiation is nonnegligible. We also note the influence of the incident angle $\theta$ on the collimated part with a decrease in light penetration with the increase in the incident angle (the diffuse radiation is here assumed to have an isotropic angular distribution on the illuminated surface). Like the degree of collimation of the radiation, this will influence cultivation system efficiency. An example of light attenuation profile is given in Fig. 26.3. Both collimated and diffuse radiations contribute to the resulting fluence rate field (for a more detailed description see again Pruvost et al. [20]).

\subsubsection{The Specific Rate of Photon Absorption and the Working Illuminated Fraction $\gamma$}

The fluence rate distribution allows determining two significant parameters to be determined: the specific rate of photon absorption noted A (here expressed per unit of biomass, i.e., in $\mu \mathrm{mol}_{\mathrm{h \nu}} \mathrm{s}^{-1} \mathrm{~kg}^{-1}$ ) and the illuminated fraction $\gamma$ (dimensionless).

Surprisingly, even though the rate of photon absorption has been found beneficial in numerous studies devoted to photoreaction, it is rarely used in microalgal culture [52]. A is obtained by the product of local fluence rate $G$ and microalgae mass absorption coefficient $E_{\mathrm{a}}$ (see previously). This value has been demonstrated as useful in photoreactor or PBR modeling $[39,53,54]$, to relate light absorption conditions to (biological) reactions. The rate 


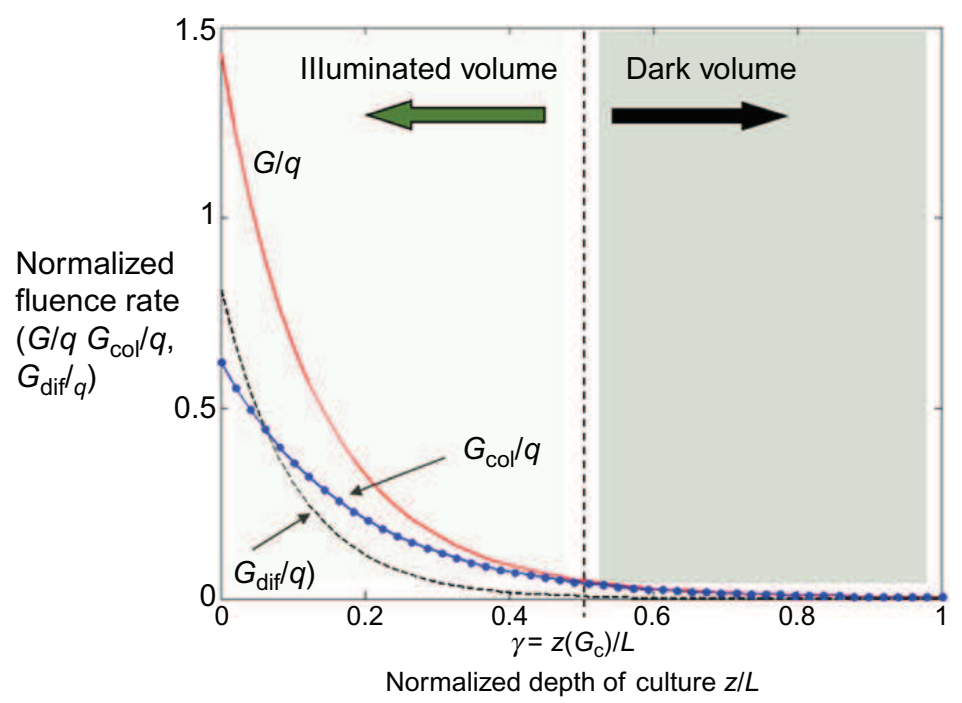

FIG. 26.3 Example of fluence rate field in bulk culture (solar radiation, full light absorption).

of photon absorption represents the light effectively absorbed by the cells, which is a combination of light received (irradiance $G$ ) and the ability of the cells to absorb light (absorption coefficient $E_{\mathrm{a}}$ ). As light absorption by cells depends of their pigment content, which is highly variable, rate of photon absorption was found as a more representative quantity to relate light effect on both kinetics and cells regulation mechanisms (than considering the fluence rate value alone). We can cite here Soulies et al. [55] who investigated the influence of specific lighting conditions such as a change in light spectrum or incident angle. Introducing specific rate of photon absorption A was found useful to relate these conditions to growth kinetics and thus make it possible to capture the respective influences of absorption rates and growth of red and white lights and nonnormal incident angles. Those authors also reported marked pigment acclimation in the studied strain (i.e., Chlorella vulgaris) which tended to compensate the fast decrease in available light with culture depth (in the case of red light) but also nonnormal incident angle. The rate of photon absorption was found to help efficiently represent all effects and was then proven as a value of interest in microalgal culture optimization. Note that another example will be given later for lipids production.

The illuminated fraction $\gamma$ was also introduced as a simple parameter to optimize light attenuation conditions in microalgal culture systems $[1,36,56,57]$. Schematically, the culture bulk can be delimited into two zones, an illuminated zone and a dark zone (Fig.26.3). Partitioning is obtained by the compensation fluence rate value $G_{c}$ corresponding to the minimum value of radiant energy required to obtain a positive photosynthetic growth rate. For example, compensation fluence rates $G_{\mathrm{c}}=1.5 \mu \mathrm{mol} \mathrm{m}^{-2} \mathrm{~s}^{-1}[36]$ and $G_{\mathrm{c}}=10 \mu \mathrm{molm}^{-2} \mathrm{~s}^{-1}$ [50] were found for Arthrospira platensis and Chlamydomonas reinhardtii, respectively. The illuminated fraction $\gamma$ is then given by the depth of the culture $z_{\mathrm{c}}$ where the fluence rate of compensation $G\left(z_{c}\right)=G_{c}$ is obtained (Fig. 26.3).

In the case of cultivation systems with one-dimensional light attenuation, we have.

$$
\gamma=\frac{V_{\mathrm{i}}}{V_{\mathrm{r}}}=\frac{z_{\mathrm{c}}}{L}
$$


where $V_{\mathrm{i}}$ and $V_{\mathrm{r}}$ are the illuminated and total culture volumes, respectively.

Values of $\gamma$ below 1 indicate that all the available light for photosynthesis received is absorbed by the culture. Conversely, when the illuminated fraction is $>1$, some of the light is transmitted (kinetic regime). This value has been shown to directly influence the performance of any light-limited biomass production [36, 50]. Because it does not allow full absorption of the light captured, the kinetic regime always leads to a loss of efficiency $(\gamma>1)$. Full light absorption is thus to be preferred $(\gamma \leq 1)$, with, however, a negative influence of the dark zone for microorganisms presenting respiration in light, such as microalgae (see below).

\subsubsection{Biomass Productivity Determination}

Biomass productivity $P_{x}$ is usually expressed in terms of volumetric productivity $\left(\mathrm{kg} \mathrm{m}^{-3} \mathrm{~h}^{-1}\right)$. In the context of mass scale production, surface productivity $\left(S_{x}, \mathrm{~kg} \mathrm{~m}^{-2} \mathrm{~h}^{-1}\right)$ is also a useful variable to extrapolate to land area production. It has also been shown that maximal performance of a cultivation system (in light-limited conditions) when expressed on a surface basis is independent of the cultivation system design [32, 48]. Both volumetric and surface productivities are linked in the following relation:

$$
S_{X}=\frac{P_{X} V_{\mathrm{r}}}{S_{\text {light }}}=\frac{P_{X}}{a_{\text {light }}}
$$

This equation introduces the specific illuminated surface $a_{\text {light }}$, which represents the illuminated surface $\left(S_{\text {light }}\right)$ to volume $\left(V_{\mathrm{r}}\right)$ ratio of the cultivation system.

In continuous mode, the biomass volumetric productivity $P_{x}$ is obtained for a given residence time $\tau$ (or dilution rate $D=1 / \tau$ ) by measuring the biomass concentration $C_{x}$ inside the cultivation system.

$$
P_{x}=D \cdot C_{X}=\frac{C_{X}}{\tau}
$$

We note that in the case of a steady state continuous production $\left(\mathrm{d} C_{x} / \mathrm{d} t=0\right)$, the biomass volumetric productivity is equal to the mean biomass volumetric growth rate in the cultivation system $\left(P_{x}=<r_{x}>\right)$.

For a batch culture, the mean biomass volumetric productivity can be estimated from the culture duration $t_{\mathrm{c}}$ before harvesting.

$$
\overline{P_{X}}=\frac{C_{X}-C_{X 0}}{t_{c}}
$$

where $C_{X 0}$ is the initial biomass concentration.

Biomass productivity can be obtained experimentally by direct measurement of the biomass concentration [50], or theoretically by solving Eq. (26.3) (here in light-limited conditions) in combination with an appropriate formulation of kinetic growth (Eqs. 26.4 and 26.5) and radiative transfer in the culture bulk (Eqs. 26.6-26.8). This involves integrating the volumetric growth rate $r_{x}$ over the reactor volume, because the heterogeneous distribution of the fluence rate field makes growth rate a local value. This integration enables us to determine the mean volumetric growth rate $\left\langle r_{x}\right\rangle$ to solve Eq. (26.3). 


$$
\left\langle r_{x}\right\rangle=\frac{1}{V_{\mathrm{r}}} \iiint_{V_{\mathrm{r}}} r_{x} \mathrm{~d} V
$$

For a cultivation system with one-dimensional light attenuation, this consists in a simple integration along the depth of culture $z$.

$$
\left\langle r_{x}\right\rangle=\frac{1}{L} \int_{0}^{L} r_{x} \cdot \mathrm{d} z
$$

where $L$ is the photobioreactor depth.

For a given species (characterized by its optical properties and kinetic growth parameters), biomass productivity will be a function of cultivation system engineering (especially the depth of culture) and operating parameters such as the dilution rate $D$ (or residence time $\tau$ ) or incident PFD. As a result, biomass productivity is difficult to predict in a simple manner. This makes the theoretical approach of prime relevance to predicting productivity evolution as a function of these key parameters and thus to photobioreactor optimization.

\subsection{PRODUCTIVITY OF MICROALGAL CULTIVATION SYSTEMS}

\subsubsection{Main Limiting Parameters Affecting Productivity}

Assuming that culture conditions ( $\mathrm{pH}$ and temperature) are kept optimal, light, carbon, and mineral nutrient supplies are the main variables liable to limit photosynthetic growth and thereby reduce the productivity of cultivation systems (assuming there are no predatory contaminations). As discussed below, nutrient and $\mathrm{CO}_{2}$ limitations can be avoided, but not light limitation, because of light attenuation in culture and of the high light requirement for photosynthesis. This simple but important observation is central to the optimization of microalgal cultivation systems. One major consequence will be the need to develop specific geometries maximizing light supply to the culture.

\subsubsection{Nutrient and Carbon Source Limitation}

To prevent mineral limitation, the growth medium must contain all the necessary nutrients (macro and micro) in sufficient quantities and must therefore, be adjusted according to the biomass concentration planned. Stoichiometric Eqs. (26.1) and (26.2) can be used for this purpose, or more simply, concentration monitoring during cultivation. The reader can also refer to studies in which the method has been applied to various species [14, 32]. In specific cases, it would also be of interest to apply mineral limitation to induce specific metabolic responses, such as lipid accumulation (nitrogen source deprivation) or hydrogen production (sulfur deprivation). Stoichiometric equations can obviously be used for this purpose (e.g., the interested reader can see Pruvost et al. [32]).

As the inorganic carbon source comes ideally from $\mathrm{CO}_{2}$ dissolved in the culture medium, preventing carbon limitation is more problematic. It depends on the gas-liquid mass transfer rate and the dissolved carbon concentration obtained. $\mathrm{CO}_{2}$ dissolution also affects the $\mathrm{pH}$ value (acidification), which in turn influences the amount and form of dissolved carbon 
obtained $\left(\mathrm{CO}_{2}, \mathrm{HCO}_{3}^{-}\right.$or $\left.\mathrm{CO}_{3}^{2-}\right)$. As stated above, nutrient consumption can also interact with $\mathrm{pH}$ evolution. Keeping an optimal $\mathrm{pH}$ value for growth, while averting limitation by the carbon source may thus not be trivial. However, in most cases, simple $\mathrm{CO}_{2}$ bubbling is usually found to suffice in the first instance for both $\mathrm{pH}$ regulation (acidification) and carbon feeding. In specific cases however, such as when using an ammonium source (the consumption of which also leads to acidification), this could be more difficult. The dissolved carbon concentration can always be monitored experimentally to forestall limitation [1].

\subsubsection{Achieving Maximal Productivities}

The growth of photosynthetic microorganisms depends on various parameters. Culture conditions ( $\mathrm{pH}$ and temperature) can be kept optimal by appropriate regulation, although at large scale and in external solar conditions this can be very difficult (see later for the case of temperature). Chemical nutrients (inorganic dissolved carbon and mineral nutrients) can be supplied, while avoiding limiting or toxic concentrations. If all parameters are kept at their optimal value and nutrients are given in adequate quantities, light-limited conditions where light alone limits growth will be achieved. By definition, this will allow maximal biomass performance.

As recently discussed and clarified elsewhere, the light-limited regime is not sufficient to obtain maximal biomass productivities. This implies additionally controlling the radiative transfer conditions inside the culture, as represented by the $\gamma$ parameter $[32,36,50]$. If the biomass concentration is too low, some of the light is transmitted through the culture (low absorption, favoring the "kinetic" regime). Conversely, if the biomass is too high, a dark zone appears deep in the culture (favoring the light-limitation regime). A distinction must be made here between eukaryotic (microalgae) and prokaryotic (cyanobacteria) cells. In the case of cyanobacteria cultivation, having common electron carrier chains and no short-time respiration in the dark [37], a dark zone will be sufficient $(\gamma \leq 1)$ to guarantee maximal productivity $[36,48]$. For eukaryotic cells presenting respiration in the light (microalgae), a dark zone in the culture volume where respiration is predominant will result in a loss of productivity due to biomass catabolism. Maximal productivity will then require the $\gamma$ fraction to meet the exact condition $\gamma=1$ (the "luminostat" regime), corresponding to a full absorption of the light received but without a dark zone in the culture volume [50].

In practice, maintaining an optimal value of the $\gamma$ parameter is not easy, especially in the case of microalgae (which implies meeting the condition $\gamma=1$ ). Some illustrations are given below for both batch and continuous production modes. Because it does not allow full absorption of the light captured, the kinetic regime always leads to a loss of efficiency $(\gamma>1)$. This regime is, however, usually encountered at the beginning of a batch production run (Fig. 26.4). Because of the biomass growth, attenuation conditions will continuously evolve and the $\gamma$ value will progressively decrease down to a value below 1 . For prokaryotic cells (Fig. 26.4, left), as soon as full absorption is obtained, the maximal value of the mean volumetric growth rate will be achieved and then remain constant (until a large dark zone is formed, inducing a shift in the cell metabolism). For eukaryotic cells, the $\gamma=1$ condition, and so the maximal value of the mean volumetric growth rate will only be transitorily satisfied (mean volumetric growth rate being represented by the slope of $C_{x}(t)$, see Eq. 26.3). 


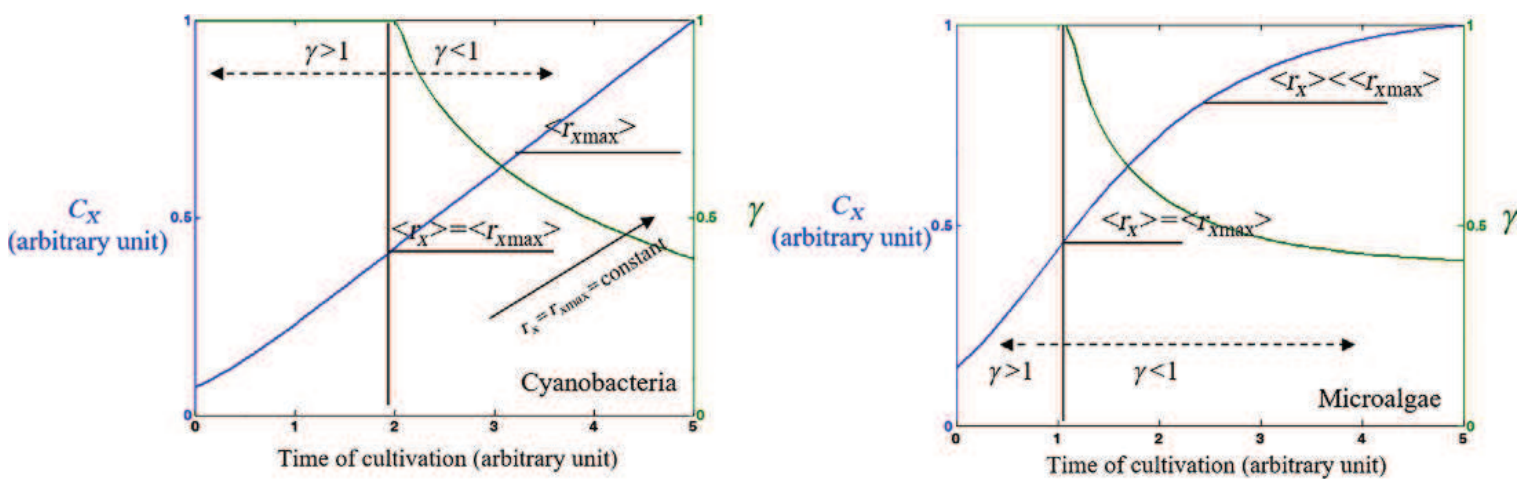

FIG. 26.4 Typical evolution of biomass concentration during a batch cultivation of cyanobacteria (left) and microalgae (right) (light-limited conditions).

The increase in the dark volume will then progressively lower the mean volumetric growth rate (Fig. 26.4, right).

In continuous mode, light attenuation conditions can be controlled by modifying the dilution rate to adjust biomass concentration in the system. For cyanobacteria (Fig. 26.5), there will be an optimal range of biomass concentrations to meet the condition $\gamma \leq 1$. For microalgae, the $\gamma=1$ condition will require an optimal biomass concentration $\left(C_{x \text { opt }}\right)$ corresponding exactly to the occurrence of the physical limitation by light, with all light absorbed but no dark zone (as shown in Takache et al. [50], a deviation of the $\gamma$ value in the range $\gamma=1 \pm 15 \%$ could be tolerated).

Whichever the production mode (continuous or batch), the control of the illuminated fraction in light limited-conditions (with $\gamma \leq 1$ for cyanobacteria and $\gamma=1 \pm 15 \%$ for microalgae) will enable us to obtain maximum biomass productivity of the cultivation system in lightlimited conditions (volume and surface). If radiative transfer conditions are known (using a radiative transfer model, as already described) then the optimal biomass concentration
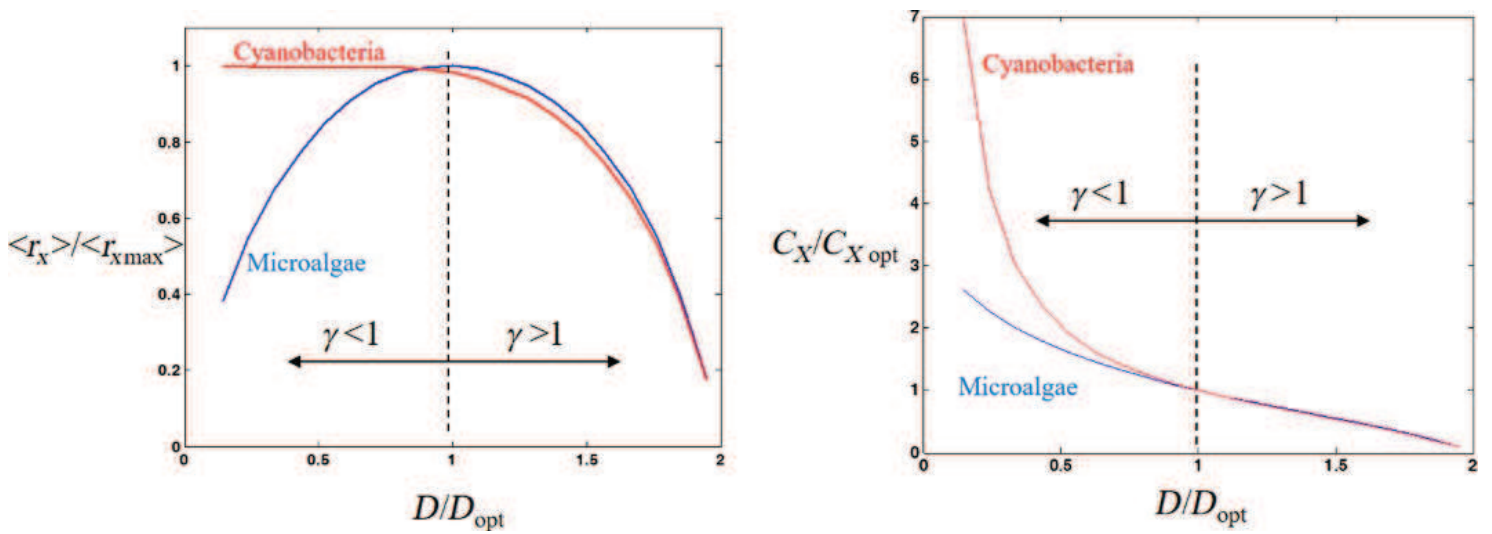

FIG. 26.5 Typical evolution of biomass volumetric productivity (left) and biomass concentration (right) as a function of the dilution rate for both cyanobacteria and microalgae (continuous production in light-limited conditions). 
can be sought theoretically. But experimental determination is also possible simply by varying the dilution rate and measuring corresponding biomass concentration and productivity [50].

\subsection{ENGINEERING PARAMETERS GOVERNING PHOTOBIOREACTOR PRODUCTIVITY}

\subsubsection{Optimization of the Light Supply}

It is well established that cultivation of photosynthetic microorganisms is highly dependent on the light supply, especially in light-limitation conditions. The light supply can be increased either by increasing the PFD or by increasing the specific illuminated surface $a_{\text {light }}$ (illuminated surface to culture volume ratio). Working in light-limited conditions with full light absorption is again important when increasing the PFD. The mutual shading of cells, in combination with adequate mixing conditions, will largely prevent photoinhibition effects [12]. This enables us to work up to very high PFD $\left(1000 \mu \mathrm{mol} \mathrm{m}{ }^{-2} \mathrm{~s}^{-1}\right.$ and above, see Ref. [50]) significantly higher than the maximum value that can be supported in dilute culture, as usually represented by the fluence rate of saturation $G_{S}$ (usually in the range 200-500 $\mu \mathrm{mol} \mathrm{m}^{-2} \mathrm{~s}^{-1}$ ).

The relation between biomass productivities and PFD was recently introduced by Cornet and Dussap [36], who proposed a simple relation. This relation was determined for cultivation systems working in light-limited conditions meeting the condition $\gamma=1$ (luminostat regime) and for geometries coming under the one-dimensional hypothesis (flat panel geometries, open ponds, and cylindrical and tubular photobioreactors with radial illumination). This relation has since been validated on a large number of photobioreactor geometries and species, including microalgae and cyanobacteria [32, 36, 50].

The equation for calculating maximal biomass volumetric productivity in light-limited conditions is.

$$
P_{x \max }=<r_{X}>_{\max }=\rho_{\mathrm{M}} \bar{\varphi} \frac{2 \alpha}{1+\alpha} a_{\text {light }} K \ln \left[1+\frac{q}{K}\right]
$$

All the parameters can be determined predictively for any species or cultivation systems geometry (for details, see Cornet and Dussap [36]). The parameters $\rho_{\mathrm{m}}, \bar{\varphi}, K$, and $\alpha$ (linear scattering modulus $\left.\alpha=\sqrt{E_{\mathrm{a}} /\left(E_{\mathrm{a}}+2 b E_{\mathrm{s}}\right)}\right)$ are species dependent. The specific illuminated surface $a_{\text {light }}$ and the PFD $q$ are engineering parameters.

Due to the progressive saturation of photosynthesis with respect to light received, increasing the PFD increases volumetric productivity, but with a progressive decrease in the yield of light conversion into biomass. This results in a logarithmic relation between the productivity and the PFD. Biomass volumetric productivity is by contrast found to increase proportionally with the specific illuminated surface $a_{\text {light }}$, emphasizing the utility of maximizing illuminated surface with respect to culture volume. For example, for Cartesian geometries (this includes flat panel photobioreactors and also open ponds), the $a_{\text {light }}$ value is directly related to the depth of culture $L_{z}$ by the simple relation $a_{\text {light }}=S_{\text {light }} / V_{\mathrm{r}}=1 / L_{z}$. Very high volumetric productivities will thus be obtained for technologies with very short light paths. Depths of 
culture are usually in the range of $0.1 \mathrm{~m}$ (with depths up to $0.5 \mathrm{~m}$ for open ponds), but values below $0.01 \mathrm{~m}$ can be also encountered. Considering Eq. (26.15), biomass volumetric productivity will then be increased 100-fold. In practice however, very narrow light paths induce specific constraints, such as difficulty maintaining adequate heat and mass transfer conditions, or possible biofilm formation.

Eq. (26.15) can also be expressed in terms of maximum surface biomass productivity.

$$
<S_{x}>_{\max }=\frac{\left\langle r_{x \max }\right\rangle}{a_{\text {light }}}=\rho_{\mathrm{M}} \bar{\varphi} \frac{2 \alpha}{1+\alpha} K \ln \left[1+\frac{q}{K}\right]
$$

We observe that surface productivity is independent of the specific illuminated surface. This is also an important conclusion. As the specific illuminated surface is fully dependent on cultivation system geometry, surface productivity is useful for comparing efficiencies of different cultivation systems. More interestingly, it emphasizes the fact that volumetric productivity can be increased while keeping surface productivity constant (assuming that the system remains in light-limited conditions). This conclusion is of particular interest in the context of solar production of biomass for energy production uses, where surface productivity is crucial and so has to be kept maximal.

\subsubsection{Influence of Mixing Conditions}

Except for immobilized cells (not discussed here), culture mixing will be necessary not only for mass (nutrients, gas-liquid transfer) and heat transfers (temperature homogenization) but also to prevent sedimentation and biofilm formation [44, 58, 59]. In addition to these classical features of any bioreactor, mixing conditions also result in what are known as light-dark (L/D) cycle effects widely described in the literature [9, 21, 31, 60, 61]. Cells moving in the heterogeneous radiation field experience a particular history with respect to the light they absorb, composed of variations from high fluence rate level (in the vicinity of the light source) to low or quasi-null values (deep in the culture) if biomass concentration is high (Fig. 26.6).

The exact effects on the resulting growth remain to be researched. Photosynthetic conversion is indeed a dynamic process, and the fluctuating light history induced by flow can modify instantaneous conversion rates of absorbed light. However, it is very difficult to
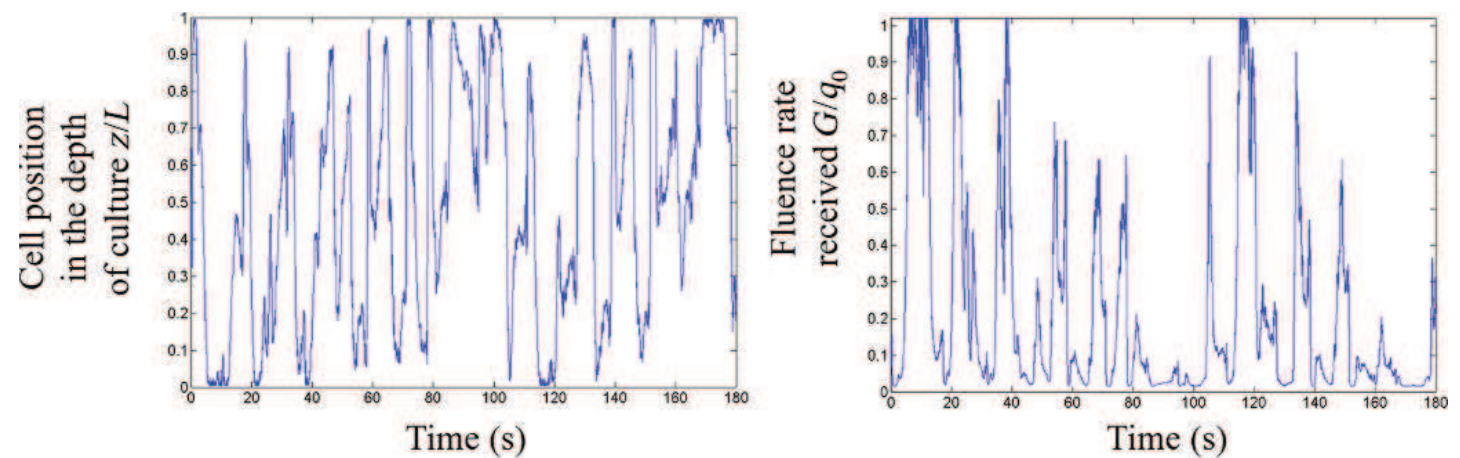

FIG. 26.6 Example of cell displacement along the light gradient (left) and corresponding light/dark cycles (right). 
investigate those effects experimentally in cultivation systems, because of various mixing effects, such as transfer enhancement (positive effect) or shear-stress generation (negative effect). Separating the coupling between the flow field and the light use from other possible mixing effects is difficult to achieve experimentally [62]. In addition, L/D cycle effects are fully dependent on the light regime, and thus on cycle frequencies and magnitudes. In cultivation systems such values are rarely known, cell history with respect to light resulting from both flow and radiative fields, each determination being a problem on its own. Some examples can be found in the literature on the characterization of light regimes in photobioreactors. Firstly, cells trajectories are determined by using either a schematic representation of the flow $[63,64,64 \mathrm{a}]$, by experimental measurement with radiative particle tracking [63, 64b], or by a Lagrangian simulation [44]. Light regime is next obtained by introducing the light attenuation model. As shown in Pruvost et al. [31], attention must, however, be paid to the formulation of the coupling. Mixing can influence the spatial distribution of particles participating in radiative transfer, resulting in a modification of the radiation field [52]. The calculation method for the radiative transfer has thus to be modified to take into account the effect of mixing conditions. An over-simplified formulation (as usually produced), where cell trajectories and radiative transfer are solved independently, results in a false representation of light availability in the reactor. This can lead for example to a significant overestimation of the L/D cycle effects [31].

In addition to the difficulty in accurately determining $\mathrm{L} / \mathrm{D}$ cycle regimes experienced by flowing cells, the corresponding biological response still remains to be clarified. It is difficult to measure and the results depend on the species and the light fluctuation magnitude and frequencies applied $[21,65]$. To characterize the effect of given mixing conditions on the system efficiency, an appropriate model has also to be formulated and then be associated with the L/D cycle prediction. Some attempts can be found in literature [63, 64, 66-71] but a research effort is still needed to develop robust and generalizable dynamic models able to represent effects of the wide range of L/D cycles encountered in microalgal cultivation system.

More vigorous mixing conditions may also have a negative effect due to the resulting hydrodynamic shear stress. Numerous species are shear stress-sensitive, with various responses, ranging from modified cell response (secretion of exopolysaccharides) to cell impairment and death [72]. A compromise has thus to be found when mixing rate is increased [73]. However, again, very few quantitative data are available, and mixing conditions, despite their influence on cultivation systems, are usually managed empirically.

In conclusion, although many studies have shown the relevance of mixing conditions, knowledge is still insufficient and useful engineering rules have yet to be found to determine optimal conditions for a given species and cultivation system. Hydrodynamic conditions have indeed several impacts that have in fine to be related. For example, fast L/D cycles with frequencies higher than $1 \mathrm{~Hz}$ are known to have a positive effect. Such frequencies can be reached in specifically designed cultivation devices [61,74]. However, this improvement will also increase energy consumption and induce shear stress. Ideally, each effect has thus to be taken into account for global optimization. But this requires a significant research effort to set up the appropriate theoretical framework necessary for systematic optimization. Actually, only general rules can be currently used to guide mixing conditions in microalgae cultivation systems. Their general objectives will be to prevent cell sedimentation, guarantee medium 
homogenization (temperature, $\mathrm{pH}$, nutrients), promote $\mathrm{L} / \mathrm{D}$ cycles by generating cell displacement along the light gradient, and keep shear stress below cell fragility thresholds.

\subsubsection{Inducing Nutrient Limitation to Produce Oil-Enriched Microalgae}

Some oleaginous species can accumulate large amount of intracellular lipids, and especially neutral lipids such as triacylglycerols (TAGs) that can be converted to biodiesel via a simple transesterification reaction $[74 \mathrm{a}, 74 \mathrm{~b}]$. Due to their significant metabolic flexibility, it is also possible to stimulate the intracellular accumulation of TAG in microalgae by varying the culture conditions. For example, nutrient stress such as nitrogen deficiency can trigger the accumulation of large amounts of neutral lipids in the form of TAGs [6, 53, 74b, 75].

The nitrogen starvation of N. oculata cultures can be achieved by two methods. Sudden starvation consists of two steps: first, microalgae are grown in nitrogen-replete conditions. Then, they are transferred into a nitrogen-free medium. Progressive starvation consists of initially adding a small amount of nitrogen to the culture medium in the form of nitrate, for example. After inoculating the PBR, the microalgae grow and multiply until they consume all the nitrates in the medium and the culture medium becomes deprived of nitrogen. Both protocols were extensively studied in Van Vooren et al. [75]. For large-scale culture, only the progressive starvation protocols seem feasible. Biomass from a culture system in nutrient replete conditions can be used, for example, to inoculate a second system operating in batch mode where nitrogen consumption during initial growth initiates starvation promptly. However, this resulted in a fully dynamic process, as several linked key-parameters will present time evolutions (biomass and nitrogen concentrations and also pigment contents that are highly impacted by nitrogen deprivation). In solar culture, this should remain challenging because of the additional effect of diurnal cycles [76].

Furthermore, Kandilian et al. [77] have shown the direct relation of the specific rate of photon absorption with lipid accumulation in the condition of nitrogen starvation. This quantity was found relevant to relate the decrease the pigment content, thus altering light absorption by cells. The authors found that a given value of specific rate of photon absorption A was necessary to trigger TAG over-accumulation and the TAG synthesis rate was also strongly related to A. This result should then be considered for the development of optimized procedure for TAG-enriched microalgae production in outdoor solar conditions.

\subsection{EXISTING TECHNOLOGY}

\subsubsection{Microalgae Culture Systems}

Unlike other more classical bioprocesses where mixing tanks essentially have standard geometries, microalgal cultivation is characterized by a broad diversity of systems, ranging from open ponds (open systems) to photobioreactor (PBR) technologies (closed systems). Detailed descriptions of existing geometries can be found in the literature [10-13]. The interested reader can also refer to two recent author's reviews [78, 79]. Following sections will give the main features of outdoor microalgal culture. 


\subsubsection{Specific Features of Solar Cultivation Systems}

Microalgal cultivation systems can use artificial or natural (sun) light sources. Obviously, for practical, economic, and environmental reasons, natural sunlight is to be preferred for mass-scale production of biomass for energy production purposes. This case will be explored here.

Whatever the technology (open or closed technologies), optimal operating in real outdoor conditions is challenging. This especially concerns (i) culture contamination issues, (ii) the optimization of the light conversion by the culture to achieve maximal performances of the system, and (iii) the difficulty to maintain optimal growth conditions such as temperature of culture [9, 20, 27, 80].

Regarding light conversion optimization, solar production adds a degree of complexity to the optimization and control of the cultivation system, compared with the artificial illumination case. The process is fully dynamic and driven by an uncontrolled input: the solar incident flux. Sunlight is highly variable in time (day-night cycles, season, and clouds) and space (Earth location, orientation of the cultivation system with respect to the sun path, shading by surrounding buildings, trees or others cultivation systems, etc.). All these features already affect more classical solar processes such as photovoltaic panels, solar thermal concentrated conversion or photocatalysis [24]. Microalgae production involves in addition specific features such as the need to keep growth conditions in an acceptable range (thermal regulation will thus be of prime relevance), or the complex biological response to light (e.g., saturation or photoinhibition effects) and dark (biomass catabolism at night). Unlike processes based only on surface conversion (e.g., photovoltaic panels), optimizing the amount of light collected on the microalgal cultivation system surface is thus not sufficient. Light conversion by photosynthetic microorganisms occurs within the culture bulk: the transfer of the collected light flux inside the bulk has thus to be taken into account. As a consequence, as for any light-driven process, cultivation systems will be highly dependent on the light collected on the illuminated surface, but light transfer conditions and thus productivity will be also influenced by the variation of incident angle or direct/diffuse distribution of sunlight flux density. All these aspects formed the subject of different author's papers [20, 26, 27] introducing a generic model to represent light-limited growth in solar photobioreactors (based on the theoretical framework presented in previous sections, drawn from many years of investigation in artificial light systems). The model was associated in particular with a solar database to predict surface productivity as a function of the system location or its ability to intercept solar radiation (e.g., as influenced by system inclination or season).

One main consequence of working in solar conditions is the dynamic regime imposed by radiation variations (e.g., day-night cycle). Transient behavior is obtained as a result of a complex interaction between physical (light) and biological (growth) kinetics, with a specific role of night, which induces biomass catabolism. The marked, steep changes in radiation conditions during the day hinder the overall optimization of the process. Whereas in artificial light the PFD can be kept constant allowing an optimized biomass concentration to obtain maximal performance from the cultivation system, in sunlight, nonideal illumination conditions prevail most of the time due to the low growth rate of algae: at noon, biomass concentration will not increase sufficiently fast to ensure full absorption of impinging light and the beginning and end of day, dark zones will appear in the culture bulk [27]. All these features are 


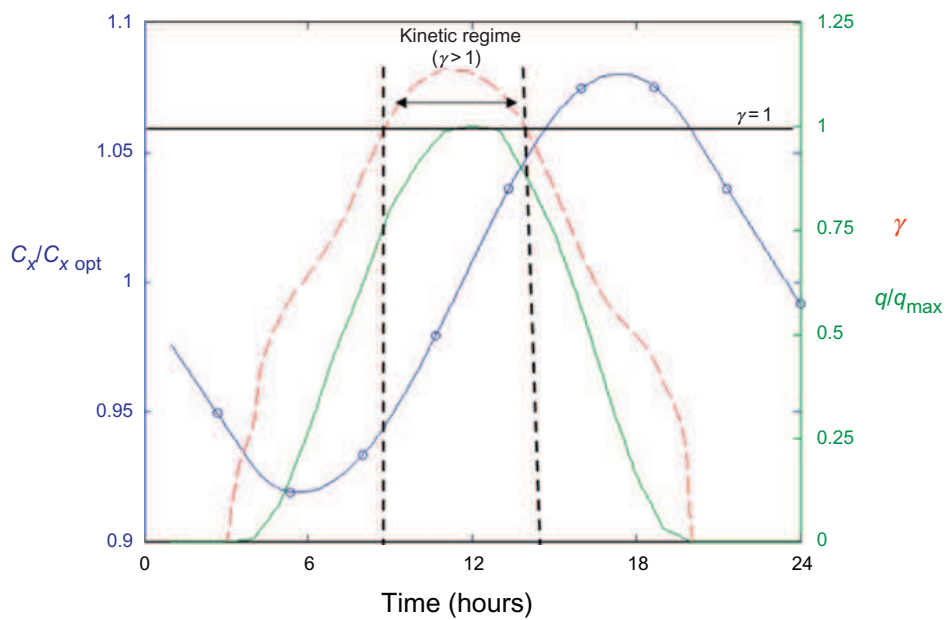

FIG. 26.7 Typical day-night variation of biomass concentration (circles added on lines) and illuminated fraction (dashed line) in a surface-lightened photobioreactor during a summer day. The normalized PFD (solid line) is also given.

characterized by the illuminated fraction, which always varies in solar conditions (Fig. 26.7). Control strategies can be devised to optimize light use during day-night cycles, such as with the harvesting procedure to optimize biomass concentration in the system and thus in the illuminated fraction for given period of the day. However, the high variability of sunlight makes this very difficult (besides day-night cycles, weather conditions have also to be allowed for) and the species cultivated will also greatly influence the strategy (especially when cultivating eukaryotic microorganisms that are sensitive to dark zones). The growth kinetics of biomass being also slow compared with the rapid variations in incident sunlight intensity, a luminostat regime cannot be reached. At best, a compromise needs to be found to determine the conditions approaching this ideal set point for most of the day. Whatever the case, light attenuation within the culture is not easy to determine. To do so, light transfer modeling is essential [26, 39, 45, 47, 56, 81, 82]. Furthermore, it can be associated with kinetics models of photosynthetic growth for a complete representation of the cultivation system characterized by its biomass concentration and biomass productivity. The reader is referred to the following studies for further information [36, 48, 57, 81].

Similar conclusions can be drawn for other growth parameter, such as temperature. Some $50 \%$ of the energy content of solar radiation lies in the infrared spectrum (higher than PAR wavelengths). Solar technology, and especially closed systems, thus tends toward overheating (or evaporation of water in open systems) under high light flux (depending obviously on the ambient conditions). An example is given in Fig. 26.8 for a flat panel photobioreactor operated without thermal regulation in the South of France in the month of July (unpublished results). A temperature of $340 \mathrm{~K}$ is reached here $\left(67^{\circ} \mathrm{C}\right)$, obviously incompatible with a microalgal cultivation. The control of temperature is thus a further challenge for mass-scale production, especially in the case of an energy-production end use. The energy balance of the process being of prime relevance, energy consumption for thermal regulation has thus to be minimized and kept in an acceptable range (at least below the energy recovered in biofuel). This implies appropriate engineering of the system but, again, the problem is not trivial, the thermal behavior (depending on the light flux) directly influencing the biomass growth (the appropriate temperature window is strongly dependent on species cultivated but typically in the $10-30^{\circ} \mathrm{C}$ range). 


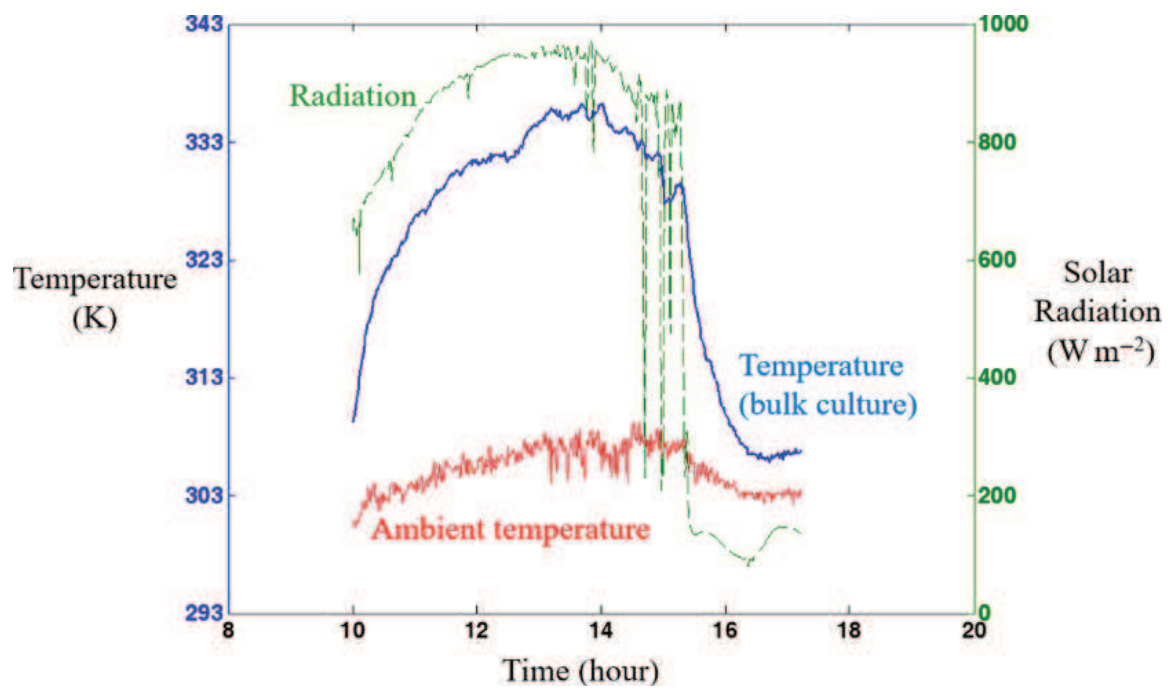

FIG. 26.8 Typical thermal behavior of a flat panel system during a sunny day in France (Perpignan, July 2010). To emphasize the overheating, the system was operated without thermal regulation (water solution and black dye).

As a major issue of solar microalgal cultivation, temperature and thermal regulation of microalgal culture systems has been widely investigated, and the interested reader can refer to literature for more details [83-87]. Various solutions are available for heating or cooling microalgal culture systems depending on their design, size, and location. Water cooling and/or heating by spraying on the outside photobioreactor surfaces or by direct immersion in a pool are often used [87]. In temperate regions, cultivation systems can also be placed in greenhouses. This can be used for either open [88] or closed culture systems [89].

\subsubsection{Toward Systematic Solar Culture Optimization}

As discussed earlier, numerous features can impair outdoor microalgal production, from mineral or carbon limitation to nonideal temperature or $\mathrm{pH}$ control, non-optimized harvesting strategies, contamination, and more. There is a clear need to pursue with efforts to develop tools for solar cultivation optimization in terms of performances and culture robustness.

However, because of the rise of microalgal industry in the last few years (and research effort on microalgae-based biofuel production), several tools and models are nowadays available to deeply investigate effects of solar culture conditions. This includes outdoor research facilities (such as AlgoSolis facility, www.algosolis.com), lab-scale fully controlled photobioreactor simulating outdoor conditions, and theoretical models [90-92].

Combining modeling to experimental approaches reveals especially relevant. Whatever the operating parameter (light, temperature, $\mathrm{pH}$, etc.), mathematical modeling of the solar production case is indeed certainly at least as important as technological development. For example, modeling enables to simulate various technologies and designs. We can cite here thermal modeling as an example [85, 93-95]. Once validated, this allows optimizing through simulations culture systems engineering and operation, and also opens the possibility to set advanced-control algorithms for optimal thermal regulation strategies (same 
applies for light conversion through light attenuation optimization). Biofuel production will certainly aim at operation throughout the entire year. Hence advanced control strategies or engineering optimization procedures are crucial to having systems operate close to their maximal performance. The utility of this approach has already been demonstrated for artificially lightened photobioreactors [96]. An adequate theoretical framework for the solar case remains to be developed. It is currently the subject of a major research effort.

\subsubsection{Surface and Volumetrically Lightened Systems}

Light energy can be supplied in two general ways, by direct illumination of the cultivation system (surface-lightened systems) or by inserting light sources inside the culture volume (volumetrically lightened systems). Most cultivation systems belong to the simpler surface-lightened group $[9,11]$. A wide variety of geometries are encountered, from open ponds to tubular or flat panel photobioreactors. An extensive literature can thus be found on these systems, showing that all of them have advantages and limits as regards control of culture conditions, culture confinement, resulting hydrodynamics conditions, ease of upscaling, construction cost, etc. However, whatever the concept, light supply and its use by the culture will always govern the productivity of the cultivation system, so that PFD and the specific illuminated surface will be the main engineering parameters. In solar conditions, the PFD is defined by the ability of the system to collect light. As for any solar processes, various positioning options are found, with systems positioned horizontally [97-99], vertically [100-102], or in few cases tilted [103, 104]. Maximizing light interception is not trivial, however. It naturally depends on the system location on the Earth and on the day or year period. For example, horizontal systems are best suited to locations close to the Equator (latitude 0 degree). For higher latitudes, it will be necessary to increase the titled angle to maximize light collection (roughly speaking, the best inclination angle for a given position on the Earth is equal to the latitude of the location). Although maximizing light intercepted must be a basic principle of any microalgal cultivation system (as for any solar process), other constraints have also to be considered. For example, using the airlift principle for mixing will preclude horizontal geometries, and shading will have to be considered when arranging vertical or tilted systems on a given land area. Again, optimizing a solar cultivation system thus proves more complex than for other classical solar processes, such as photovoltaic panels, where light intercepted is the only parameter (of a given panel technology).

Volumetrically lightened systems lead to more complex technologies, but allow a further optimization of the light use in the culture. Firstly, insertion of light sources in the culture bulk guarantees a maximal use of emitted photons. For surface-lightened systems, and especially for artificially lightened systems, it is very difficult to collimate all the emitted light onto the optical surface of a photobioreactor. Secondly and more interestingly, internal lighting allows light to be diluted. As discussed previously, increasing light leads to higher volumetric productivity, but with a progressive decrease in the conversion yield, due to photosynthesis saturation. By diluting light received in the culture volume, a high yield can be maintained. This is of particular interest for solar use and energy production 
applications. In this case, solar light is collected on a given surface (using, e.g., a parabolic device) and then transmitted to the culture (using, e.g., optical fibers). Because of the high PFD usual in solar conditions, a significant increase in surface productivity can be obtained. Furthermore, the dilution principle can be combined with a solar-tracking system, giving an additional possibility of optimization by maximizing light intercepted during the sun's travel [105]. By combining these advantages in systems with high specific illuminated surfaces, the most efficient system of light conversion into biomass can be obtained, with both high volumetric and surface productivities. A full description of such a principle has been described by Cornet [48] with a volumetrically lightened PBR based on the "DiCoFluV" concept (more details are given in next sections). Theoretically, such technology allows the highest biomass productivities permitted with algae. The author presents maximal productivities that could be achieved for both surface and volumetrically lightened systems, assuming ideal sunlight conditions when located at the Equator. For surface-lightened systems (fixed horizontal photobioreactor), a mean daily ideal value of the PFD around $1000 \mu \mathrm{mol} \mathrm{m} \mathrm{s}^{-2} \mathrm{~s}^{-1}$ was harnessed, leading to a surface biomass productivity of $100 \mathrm{t} \mathrm{ha}^{-1} \mathrm{y}^{-1}$ with an exergetic yield of the photobioreactor of $6 \%$. For volumetrically lightened systems with a sun-tracking system ("DiCoFluV" concept), the daily averaged PFD was increased to $1400 \mu \mathrm{mol} \mathrm{m}^{-2} \mathrm{~s}^{-1}$ (same equatorial location). In combination with the dilution principle, a surface biomass productivity of $400 \mathrm{tha}^{-1} \mathrm{y}^{-1}$ was obtained, corresponding to a maximum energy yield of the photobioreactor of $17 \%$. Because all the calculations were conducted here for an ideal case (solar radiation, growth kinetics, photobioreactor design, and light use), this corresponds to the upper limit of productivity that may be achieved on the Earth with photosynthetic microorganisms.

Volumetrically illuminated PBRs hold great promise, but there are still rare few examples in the literature [41, 48, 106-108], mainly due to the technological complexity involved and the difficulty scaling up PBR systems to large surface areas. Further technological developments are still needed.

\subsubsection{Open systems and Photobioreactors}

Several recent reviews on existing technologies for microalgal production can be found in the literature and only the main aspects will be given here.

The cheapest systems that can be easily extended today to a large scale are open systems. These systems have been used for many decades at an industrial scale, but for applications other than biofuels [9]. Technologically, such systems could, however, be used for that purpose. The two main groups of open systems are natural ponds and raceways. The main difference between them is in the mixing regime. Open ponds are unmixed (except naturally, e.g., by wind), unlike raceways, where paddle wheels are used to circulate the culture in a loop configuration. The best productivities are obtained in these last systems. The main limitations of open systems are inherent to their operating principle. Owing to the direct contact with the atmosphere, there is a high risk of biological contamination (other microalgae species, bacteria, predators, etc.). Only resistant species can thus be long-term cultivated in such systems. Because there is a large interface between the culture and the atmosphere, their control is also difficult, for example, to maintain optimal temperature (although open systems are less subject to overheating than 
closed systems). In addition, the gas-liquid equilibrium with the rather low atmospheric $\mathrm{CO}_{2}$ content generally results in a limiting concentration of dissolved carbon, insufficient to meet the needs of photosynthetic microorganisms in the case of intensive production. A carbon supply can be added ( $\mathrm{CO}_{2}$ or carbonate), but a significant part of this will inevitably be degassed into the atmosphere, making carbon limitation difficult to prevent entirely.

The raceway system is certainly the most widespread technology for large-scale culture and it has gained currency as a mature cultivation system $[98,109,110]$. Raceway technologies are effectively easy to scale-up and cheap to build. Different materials can be used, from clay to PVC [111]. Over the last few years, optimizations have been proposed, such as using computational fluid dynamics to optimize mixing with a paddlewheel design [112-115] to maximize biomass productivity and light distribution in the culture volume and reduce power consumption [116]. Raceways technology appears nowadays clearly as a reference for outdoor mass-scale production. However, as for any open culture system, it suffers from intrinsic limitations, such as the risk of biological contamination. It is then well-adapted to producing extremophile or resistant species. On the other hand, if the goal of the system is to produce sensitive microorganisms, then a closed system (PBR) is needed.

Closed geometries reduce risks of external contamination and a better control of culture conditions is obtained. The higher partial pressure allowed in the gas phase will also prevent carbon limitation. All these advantages will allow the light-limitation condition to be obtained and as already discussed, productivity will then be limited only by the solar energy entering the cultivation system and by its use either by direct illumination or by diluting light in the culture bulk. However, closed geometries suffer from several limitations and also inherent to their operating principles. Culture confinement increases the risk of biofilm formation, leads to oxygen accumulation in the culture (toxic effects), and overheating can occur (especially in solar conditions due to the large amount of infrared light collected). Unlike open systems where the only way to prevent external contamination or carbon limitation is to close the systems (working then with photobioreactor technology), the limits of closed geometries can be at least partly overcome by appropriate engineering of photobioreactors (e.g., by adapting mixing conditions to increase heat and gas-liquid mass transfer or to prevent biofilm formation). However, this most often also results in increased cost and complexity. As the photobioreactor is the only system that allows maximal productivity to be obtained (by working in light-limited conditions), great efforts are currently being made to develop new technology devoted to mass scale production.

The literature counts an array of reports and publications on the various PBR technologies available $[11,13,32,79,87,100,117,118]$. PBR technologies are characterized by a wide diversity including tubular, cylindrical, and flat-panel systems. This diversity of PBR designs is the result of various attempts to optimize light capture while satisfying other practical constraints related to (i) engineering design, including system integration, scale of production, materials selection, and project costs, and (ii) system operation factors such as $\mathrm{CO}_{2}$ bubbling, oxygen removals, temperature and $\mathrm{pH}$ regulation, nutrient delivery, etc.

Nowadays, current mass scale production comes mainly from extensive open systems that are easier to build and operate, that is, open ponds or raceway systems. However, it may well be that in the near future a suitable closed technology will be devised that meets the criteria for mass-scale intensified production of photosynthetic microorganisms. 


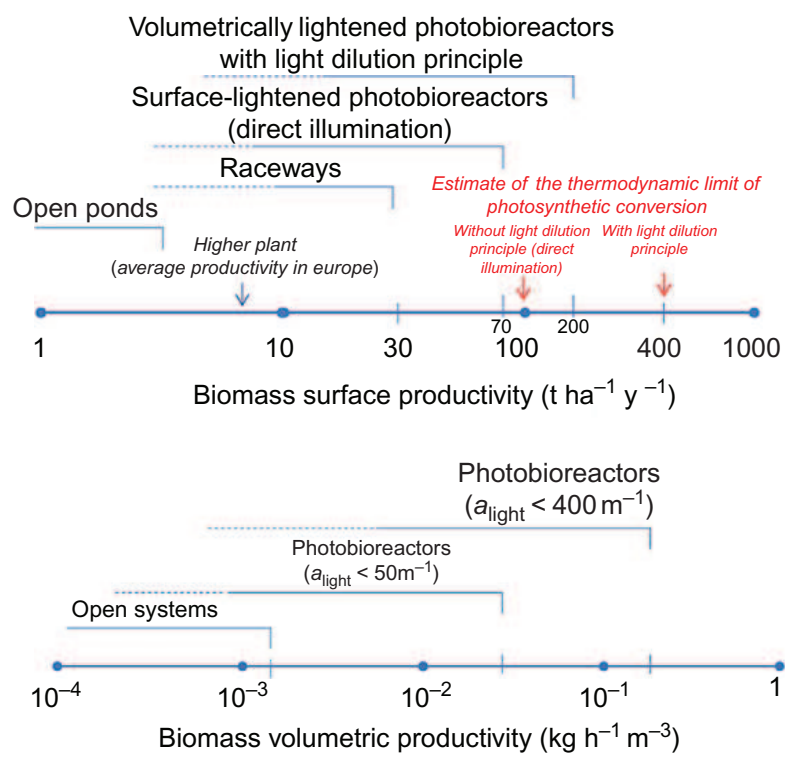

FIG. 26.9 Estimate of the maximal surface (top) and volumetric (bottom) productivities that could be achieved with different microalgal cultivation systems.

Fig. 26.9 (top) gives a rough estimate of the maximal surface productivity that could be achieved with the different technologies (all for an ideal case, as defined by Cornet [48]). The lower surface productivity of open systems is assumed here, considering the lower control of culture conditions and effect of carbon limitation, with raceways presenting higher productivities than open ponds due to the mixing optimization they permit. Higher surface productivities are obtained with volumetrically lightened photobioreactors allowing light dilution in the culture bulk to prevent from adverse effects of photosynthesis saturation to light, as encountered in surface-lightened photobioreactors having thus lower surface productivity. Fig. 26.9 (bottom) gives an overview of volumetric productivity of microalgal cultivation systems that is highly linked to their specific illuminated surface and thus culture depth. Raceway depths are usually about $0.2 \mathrm{~m}$, while photobioreactor depths can be as low as a few centimeters or even less. Using Eq. (26.15) and considering also that open systems are usually submitted to other limitations than light, the volumetric productivity of photobioreactor can be thus higher by two orders of magnitude. As already discussed, surface productivity is relevant for its direct impact on land areas required for a given production. Volumetric productivity would have also a decisive impact on the global biofuel production process. Increasing volumetric productivity will indeed allow high biomass concentration and thus lower harvesting cost and will also lower the culture volume to be managed and so energy consumption for mixing. All these aspects will contribute to a positive energy balance at the overall process level. Considering in addition that only closed systems allow carbon limitation to be prevented when working at high biomass volumetric productivity, thus leading to a higher surface productivity than in open systems, photobioreactors clearly offer the highest potential. Maximal areal productivity can be sought while increasing volumetric productivity. Limits are here mainly in engineering aspects making the development of specific cultivation systems for mass-scale production of algae at an acceptable cost as one of the main current challenges to the global use of photosynthetic microorganisms for energy production. 


\subsubsection{Interest of Photobioreactor Intensification for Biofuel Production: The AlgoFilm Concept}

As shown previously, the setting of generic engineering formula allows relating engineering parameters to resulting culture systems performances [volumetric and surface productivities, Eq. (26.15) and (26.16), respectively]. This enables developing rational approaches for process intensification. Such an approach was recently proposed by authors for the development of a solar technology named AlgoFilm [119].

The underlying concept is to increase both specific illuminated surface (by decreasing the culture depth, i.e., $a_{\text {light }}=S_{\text {light }} / V_{R}=1 / L$ for a flat panel) and PFD to increase volumetric productivity (or biomass concentration, the two being linked). More specifically, working in a thin-film (typically $\left.a_{\text {light }}>100 \mathrm{~m}^{-1}, L<0.01 \mathrm{~m}\right)$ compared to usual geometries $\left(a_{\text {light }}\right.$ around $20 \mathrm{~m}^{-1}$ for a PBR of depth $0.05 \mathrm{~m}, 0.3 \mathrm{~m}^{-1}$ for raceway of depth $0.3 \mathrm{~m}$ ) allows increasing by two orders of magnitude volumetric productivity. This makes it possible to work in high-celldensity culture $\left(C_{x}>10 \mathrm{~kg} \mathrm{~m}^{-3}\right)$ leading to the advent of high-volumetric productivity PBRs (HVP-PBR). Note too that increasing the PFD will lead to a further increase in biomass productivity (but with a decrease in thermodynamic yield of photosynthetic conversion, as previously discussed). As surface productivity is independent of specific illuminated surface (Eq. 26.16), a specific feature of PBR technology is the possibility of drastically increasing volumetric productivity while maintaining surface productivity.

This was the basic statement behind the design of AlgoFilm technology which aims to propose very high volumetric productivity (HVP-PBR) at the current performance ceiling while keeping the maximal conversion of incoming light permitted by the direct illumination principle (surface-lightened system, without light dilution). AlgoFilm PBR was based on the falling-film principle so as to obtain very thin culture (around $1.5-2 \mathrm{~mm}$ ) and provide a high specific illuminated surface area (around $500 \mathrm{~m}^{-1}$, corresponding to $2.1 \mathrm{Lm}^{-2}$ of illuminated surface). This allows achieving a biomass productivity of $5.7 \mathrm{~kg} \mathrm{~m}^{-3}$ day $^{-1}$, higher than value reported in literature (usually around $1-2 \mathrm{~kg} \mathrm{~m}^{-3}$ day $^{-1}$ for solar PBR). This value was similar to those generally found for microalgal production in heterotrophic processes $\left(5.8 \mathrm{~kg} \mathrm{~m}^{-3}\right.$ day $\left.^{-1},[120]\right)$.

The direct advantage of intensifying volumetric productivity is that it reduces the system size needed to achieve a given production requirement. In the general framework of a global industrial exploitation, energy consumption in several processes is directly linked to culture volume (pumping, mixing, temperature control, harvesting, etc.). Increasing volumetric productivity can thus drastically reduce energy needs for a given operation. This holds primary relevance in biofuel production for example, where both surface and volumetric productivities can be increased by appropriately engineering PBRs. Note that the AlgoSolis R\&D facility (www.algosolis.com) recently integrated the AlgoFilm technology as a platform for research projects in microalgal liquid biofuel production (biodiesel, biokerosene).

\subsection{CONCLUSIONS AND PERSPECTIVES}

While promising, the biofuel production from microalgae remains a major challenge. This implies to raise several obstacles, particularly related to the need to produce at large scale and 
in a sustainable way a microalgal biomass that can be converted into biofuel. This involves a joined effort both in biology (choice of effective and robust species, optimization and control of their culture at large-scale ...), and in bioprocess engineering (culture systems, downstream processing, and final conversion in biofuels).

As shown in this chapter, production alone remains a major bottleneck (downstream processing that has not been addressed in this chapter is also a major issue). The choice of culture technology influences key criteria, like biomass productivity (biomass produced per unit of land area) but also the energy consumption that can lead to a negative energy balance on its own (due to thermal regulation, or mixing). However, the main challenges are now identified and based on robust engineering approaches, innovative technologies like intensified photobioreactors and advanced control software can now be devised for an optimal solar production of microalgae. Research efforts have to be pursued and should focus on the closely connected areas of microalgal biology, engineering design, and control.

\section{Acknowledgments}

This work was supported by several projects, and especially by the French National Research Agency within the framework of the DIESALG (ANR-12-BIME-0001-02) and BIOSOLIS projects. This work is also connected to R\&D activities led at the AlgoSolis R\&D facility (www.algosolis.com). This book chapter is also the result of many years of collaboration with two remarkable scientists, Prof. Jack Legrand and Prof. Jean-François Cornet, to whom the author is especially grateful. Thanks also go to Vincent Goetz for his help on all solar aspects.

\section{References}

[1] B. Degrenne, J. Pruvost, G. Christophe, J.F. Cornet, G. Cogne, J. Legrand, Investigation of the combined effects of acetate and photobioreactor illuminated fraction in the induction of anoxia for hydrogen production by Chlamydomonas reinhardtii, Int. J. Hydrogen Energy 35 (19) (2010) 10741-10749.

[2] K. Tsukahara, S. Sawayama, Liquid fuel production using microalgae, J. Jpn. Petrol. Inst. 48 (5) (2005) 251-259.

[3] P. Spolaore, C. Joannis-Cassan, E. Duran, A. Isambert, Commercial applications of microalgae, J. Biosci. Bioeng. 101 (2) (2006) 87-96.

[4] M.L. Ghirardi, L. Zhang, J.W. Lee, et al., Microalgae: a green source of renewable $\mathrm{H}_{2}$, Trends Biotechnol. 18 (12) (2000) 506-511.

[5] Y. Chisti, Biodiesel from microalgae, Biotechnol. Adv. 25 (2007) 294-306.

[6] Q. Hu, M. Sommerfeld, E. Jarvis, et al., Microalgal triacylglycerols as feedstocks for biofuel production: perspectives and advances, Plant J. 54 (2008) 621-639.

[7] L. Rodolfi, G. Chini Zittelli, N. Bassi, et al., Microalgae for oil: strain selection, induction of lipid synthesis and outdoor mass cultivation in a low-cost photobioreactor, Biotechnol. Bioeng. 102 (1) (2009) 100-112.

[8] J.R. Benemann, Hydrogen and methane production by microalgae, in: A. Richmond (Ed.), Handbook of Microalgal Culture: Biotechnology and Applied Technology, Wiley, 2004.

[9] A. Richmond, Handbook of Microalgal Culture: Biotechnology and Applied Phycology, Oxford, UK, Blackwell Sciences Ltd, 2004.

[10] F. Lehr, C. Posten, Closed photo-bioreactors as tools for biofuel production, Curr. Opin. Biotechnol. 20 (3) (2009) 280-285.

[11] C.U. Ugwu, H. Aoyagia, H. Uchiyamaa, Photobioreactors for mass cultivation of algae, Bioresour. Technol. 99 (10) (2008) 4021-4028.

[12] A. Richmond, Principles for attaining maximal microalgal productivity in photobioreactors: an overview, Hydrobiologia 512 (2004) 33-37.

[13] A.P. Carvalho, L.A. Meireles, F.X. Malcata, Microalgal reactors: a review of enclosed system designs and performances, Biotechnol. Prog. 22 (2006) 1490-1506. 
[14] J. Pruvost, G. Van Vooren, G. Cogne, J. Legrand, Investigation of biomass and lipids production with Neochloris oleoabundans in photobioreactor, Bioresour. Technol. 100 (2009) 5988-5995.

[15] G. Cogne, B. Lehmann, C.-G. Dussap, J.B. Gros, Uptake of macrominerals and trace elements by the cyanobacterium Spirulina platensis (Arthrospira platensis PCC 8005) under photoautotrophic conditions: culture medium optimization, Biotechnol. Bioeng. 81 (5) (2003) 588-593.

[16] J.A. Roels, Energetics and Kinetics in Biotechnology, Elsevier Biomedical Press, Amsterdam, 1983.

[17] J. Doucha, F. Straka, K. Lívanský, Utilization of flue gas for cultivation of microalgae Chlorella sp. in an outdoor open thin-layer photobioreactor, J. Appl. Phycol. 17 (5) (2005) 403-412.

[18] S.Y. Chiu, C.Y. Kao, C.H. Chen, T.C. Kuan, S.C. Ong, C.S. Lin, Reduction of $\mathrm{CO}_{2}$ by a high-density culture of Chlorella sp. in a semicontinuous photobioreactor, Bioresour. Technol. 99 (2008) 3389-3396.

[19] A. Vonshak, G. Torzillo, Environmental stress physiology, in: A. Richmond (Ed.), Handbook of Microalgal Culture: Biotechnology and Applied Phycology, Blackwell Sciences Ltd, Oxford, 2004, pp. 57-82.

[20] J. Pruvost, J.F. Cornet, V. Goetz, J. Legrand, Modeling dynamic functioning of rectangular photobioreactors in solar conditions, AIChE J. 57 (2011) 1947-1960.

[21] M. Janssen, M.G.J. Janssen, M. De Winter, et al., Efficiency of light utilization of Chlamydomonas reinhardtii under medium-duration light/dark cycles, J. Biotechnol. 78 (2000) 123-137.

[22] A. Sanchez Miron, M.C. Ceron Garcia, A. Contreras Gomez, F. Garcia Camacho, E. Molina Grima, Y. Chisti, Shear stress tolerance and biochemical characterization of Phaeodactylum tricornutum in quasi steady-state continuous culture in outdoor photobioreactors, Biochem. Eng. J. 16 (3) (2003) 287-297.

[23] L. Pottier, J. Pruvost, J. Deremetz, J.F. Cornet, J. Legrand, C.G. Dussap, A fully predictive model for onedimensional light attenuation by Chlamydomonas reinhardtii in a torus reactor, Biotechnol. Bioeng. 91 (5) (2005) 569-582.

[24] J.A. Duffie, W.A. Beckman, Solar Engineering of Thermal Processes, third ed., Wiley, New York, 2006.

[25] E. Sierra, F.G. Acien, J.M. Fernandez, J.L. Garcia, C. Gonzales, E. Molina, Characterization of a flat plate photobioreactor for the production of microalgae, Chem. Eng. J. 138 (2008) 136-147.

[26] J. Pruvost, J.F. Cornet, V. Goetz, J. Legrand, Theoretical investigation of biomass productivities achievable in solar rectangular photobioreactors for the cyanobacterium Arthrospira platensis, Biotechnol. Prog. 28 (3) (2012) 699-714.

[27] J. Pruvost, J.F. Cornet, F. Le Borgne, V. Goetz, J. Legrand, Theoretical investigation of microalgae culture in the light changing conditions of solar photobioreactor production and comparison with cyanobacteria, Algal Res. 10 (2015) 87-99.

[28] Cornet J-F. Procédés limités par le transfert de rayonnement en milieu hétérogène. Etude des couplages cinétiques et énergétiques dans les photobioréacteurs par une approche thermodynamique [Habilitation à Diriger des Recherches]. Université Blaise Pascal, Clermont-Ferrand, nº d'ordre 2362007.

[29] H. Berberoglu, L. Pilon, A. Melis, Radiation characteristics of Chlamydomonas reinhardtii CC125 and its truncated chlorophyll antenna transformants tla1, tlaX and tla1-CW+, Int. J. Hydrogen Energy 33 (22) (2008) 6467-6483.

[30] J.F. Cornet, L. Favier, C.G. Dussap, Modeling stability of photoheterotrophic continuous cultures in photobioreactors, Biotechnol. Prog. 19 (4) (2003) 1216-1227.

[31] J. Pruvost, J.F. Cornet, J. Legrand, Hydrodynamics influence on light conversion in photobioreactors: an energetically consistent analysis, Chem. Eng. Sci. 63 (2008) 3679-3694.

[32] J. Pruvost, Cultivation of algae in photobioreactors for biodiesel production, in: A. Pandey, C. Larroche, S.C. Ricke, C.G. Dussap (Eds.), Biofuels: Alternative Feedstocks and Conversion Processes, InTech, 2011, pp. 439-464.

[33] S. Fouchard, J. Pruvost, B. Degrenne, M. Titica, J. Legrand, Kinetic modeling of light limitation and sulphur deprivation effects in the induction of hydrogen production with Chlamydomonas reinhardtii. Part I: Model description and parameters determination, Biotechnol. Bioeng. 102 (1) (2009) 132-147.

[34] A. Muller-Feuga, Growth as a function of rationing: a model applicable to fish and microalgae, J. Exp. Mar. Biol. Ecol. 236 (1998) 1-13.

[35] S. Aiba, Growth kinetics of photosynthetic microorganisms, Adv. Biochem. Eng. Biotechnol. 23 (1982) 85-156.

[36] J.F. Cornet, C.G. Dussap, A simple and reliable formula for assessment of maximum volumetric productivities in photobioreactors, Biotechnol. Prog. 25 (2009) 424-435.

[37] L. Gonzalez de la Vara, C. Gomez-Lojero, Participation of plastoquinone, cytochrome c553 and ferredoxin$\mathrm{NADP}+$ oxido reductase in both photosynthesis and respiration in Spirulina maxima, Photosynth. Res. 8 (1986) 65-78.

[38] Kliphuis A. Modeling of microalgal metabolism. PhD Thesis, Wageningen, The Netherlands, Wageningen University; 2010. 
[39] J. Pruvost, J.F. Cornet, Knowledge models for engineering and optimization of photobioreactors, in: C.P.A. C. Walter (Ed.), Microalgal Biotechnology, Walter de Gruyter GmbH, 2012, pp. 181-224.

[40] J.F. Cornet, C.G. Dussap, J.B. Gros, Kinetics and energetics of photosynthetic micro-organisms in photobioreactors: application to Spirulina growth, Adv. Biochem. Eng. Biotechnol. 59 (1998) 155-224.

[41] Z. Csogör, M. Herrenbauer, K. Schmidt, C. Posten, Light distribution in a novel photobioreactor-modelling for optimization, J. Appl. Phycol. 13 (2001) 325-333.

[42] M.R. Tredici, G. Chini Zittelli, Efficiency of sunlight utilization: tubular versus flat photobioreactors, Biotechnol. Bioeng. 57 (1998) 187-197.

[43] Y.S. Yun, J.M. Park, Kinetic modeling of the light-dependent photosynthetic activity of the green microalga Chlorella vulgaris, Biotechnol. Bioeng. 83 (3) (2003) 303-311.

[44] J. Pruvost, J. Legrand, P. Legentilhomme, A. Muller-Feuga, Simulation of microalgae growth in limiting light conditions-flow effect, AIChE J. 48 (2002) 1109-1120.

[45] J.F. Cornet, C.G. Dussap, P. Cluzel, G. Dubertret, A structured model for simulation of cultures of the cyanobacterium Spirulina platensis in photobioreactors. 2. Identification of kinetic parameters under light and mineral limitations, Biotechnol. Bioeng. 40 (7) (1992) 826-834.

[46] J.F. Cornet, C.G. Dussap, J.B. Gros, Conversion of radiant light energy in photobioreactors, AIChE J. 40 (6) (1994) $1055-1066$

[47] J.F. Cornet, C.G. Dussap, J.B. Gros, A simplified monodimensional approach for modeling coupling between radiant light transfer and growth kinetics in photobioreactors, Chem. Eng. Sci. 50 (9) (1995) 1489-1500.

[48] J.F. Cornet, Calculation of optimal design and ideal productivities of volumetrically-lightened photobioreactors using the constructal approach, Chem. Eng. Sci. 65 (2010) 985-998.

[49] K. Loubiere, E. Olivo, G. Bougaran, J. Pruvost, R. Robert, J. Legrand, A new photobioreactor for continuous microalgal production in hatcheries based on external-loop airlift and swirling flow, Biotechnol. Bioeng. 102 (1) (2009) 132-147.

[50] H. Takache, G. Christophe, J.F. Cornet, J. Pruvost, Experimental and theoretical assessment of maximum productivities for the micro-algae Chlamydomonas reinhardtii in two different geometries of photobioreactors, Biotechnol. Prog. 26 (2) (2010) 431-440.

[51] B. Farges, C. Laroche, J.F. Cornet, C.G. Dussap, Spectral kinetic modeling and long-term behavior assessment of Arthrospira platensis growth in photobioreactor under red $(620 \mathrm{~nm})$ light illumination, Biotechnol. Prog. 25 (1) (2009) 151-162.

[52] A.E. Cassano, C.A. Martin, R.J. Brandi, O.M. Alfano, Photoreactor analysis and design: fundamentals and applications, Ind. Eng. Chem. Res. 34 (7) (1995) 2155-2201.

[53] R. Kandilian, J. Pruvost, J. Legrand, L. Pilon, Influence of light absorption rate by Nannochloropsis oculata on triglyceride production during nitrogen starvation, Bioresour. Technol. 163 (2014) 308-319.

[54] Dauchet J. Analyse radiative des photobioreacteurs. PhD Thesis, Universite Blaise Pascal—Clermont-Ferrand, France ( $\mathrm{n}^{\circ}$ ordre 2304-in French), 2012.

[55] A. Soulies, C. Castelain, T.I. Burghelea, J. Legrand, H. Marec, J. Pruvost, Investigation and modeling of the effects of light spectrum and incident angle on the growth of Chlorella vulgaris in photobioreactors, Biotechnol. Prog. 32 (2) (2016) 247-261.

[56] J.F. Cornet, C.G. Dussap, P. Cluzel, G. Dubertret, A structured model for simulation of cultures of the cyanobacterium Spirulina platensis in photobioreactors. 1. Coupling between light transfer and growth kinetics, Biotechnol. Bioeng. 40 (7) (1992) 817-825.

[57] H. Takache, G. Christophe, J.F. Cornet, J. Pruvost, Experimental and theoretical assessment of maximum productivities for the microalgae Chlamydomonas reinhardtii in two different geometries of photobioreactors, Biotechnol. Prog. 26 (2) (2010) 431-440.

[58] A. Muller-Feuga, J. Pruvost, R. Le Guedes, L. Le Dean, P. Legentilhomme, J. Legrand, Swirling flow implementation in a photobioreactor for batch and continuous cultures of Porphyridium cruentum, Biotechnol. Bioeng. 84 (5) (2003) 544-551.

[59] A. Muller-Feuga, R. Le Guedes, J. Pruvost, Benefits and limitations of modeling for optimization of Porphyridium cruentum cultures in an annular photobioreactor, J. Biotechnol. 103 (2) (2003) 153-163.

[60] I. Perner-Nochta, C. Posten, Simulations of light intensity variation in photobioreactors, J. Biotechnol. 131 (3) (2007) 276-285.

[61] R. Rosello Sastre, Z. Csögör, I. Perner-Nochta, P. Fleck-Schneider, C. Posten, Scale-down of microalgae cultivations in tubular photo-bioreactors-A conceptual approach, J. Biotechnol. 132 (2) (2007) 127-133. 
[62] J.C. Merchuk, M. Ronen, S. Giris, S. Arad, Light/dark cycles in the growth of the red microalga Porphyridium sp., Biotechnol. Bioeng. 59 (6) (1998) 705-713.

[63] X. Wu, J.C. Merchuk, Simulation of algae growth in a bench-scale column reactor, Biotechnol. Bioeng. 80 (2) (2002) 156-168.

[64] X. Wu, J.C. Merchuk, Simulation of algae growth in a bench-scale internal loop airlift reactor, Chem. Eng. Sci. 59 (2004) 2899-2912.

[64a] M. Janssen, J. Tramper, L.R. Mur, R.H. Wijffels, Enclosed outdoor photobioreactors: light regime, photosynthetic efficiency, scale-up, and future prospects, Biotechnol. Bioeng. 81 (2) (2003) 193-210.

[64b] H.P. Luo, A. Kemoun, M.H. Al-Dahhan, J.M. Fernandez Sevilla, J.L. Garcia Sanchez, F. Garcia Camacho, E. Molina Grima, Analysis of photobioreactors for culturing high-value microalgae and cyanobacteria via an advanced diagnostic technique: CARPT, Chem. Eng. Sci. 58 (12) (2003) 2519-2527.

[65] M.G.J. Janssen, T.C. Kuijpers, B. Veldhoen, et al., Specific growth rate of Chlamydomonas reinhardtii and Chlorella sorokiniana under medium duration light/dark cycles 13-87 s, J. Biotechnol. 70 (1999) 323-333.

[66] C. Pahl-Wostl, Dynamic versus static models for photosynthesis, Hydrobiologia 238 (1992) 189-196.

[67] H.P. Luo, M.H. Al-Dahhan, Analyzing and modeling of photobioreactors by combining first principles of physiology and hydrodynamics, Biotechnol. Bioeng. 85 (4) (2004) 382-393.

[68] N. Yoshimoto, T. Sato, Y. Kondo, Dynamic discrete model of flashing light effect in photosynthesis of microalgae, J. Appl. Phycol. 17 (3) (2005) 207-214.

[69] X. Wu, J.C. Merchuk, A model integrating fluid dynamics in photosynthesis and photoinhibition processes, Chem. Eng. Sci. 56 (2001) 3527-3538.

[70] P.H.C. Eilers, J.C.H. Peeters, Dynamic behaviour of a model for photosynthesis and photoinhibition, Ecol. Model. 69 (1993) 113-133.

[71] F.R. Camacho, F.G. Camacho, F.J.M. Sevilla, Y. Chisti, E. Molina Grima, A mechanistic model of photosynthesis in microalgae, Biotechnol. Bioeng. 81 (4) (2003) 459-473.

[72] P. Jaouen, L. Vandanjon, F. Quéméneur, The shear stress of microalgal cell suspensions (Tetraselmis suecica) in tangential flow filtration systems: the role of pumps, Bioresour. Technol. 68 (1999) 149-154.

[73] M.J. Barbosa, Hadiyanto, R.H. Wijffels, Overcoming shear stress of microalgae cultures in sparged photobioreactors, Biotechnol. Bioeng. 85 (1) (2004) 78-85.

[74] M. Janssen, L. De Bresser, B. Baijens, et al., Scale-up aspects of photobioreactors: effects of mixing-induced light/dark cycles, J. Appl. Phycol. 12 (2000) 225-237.

[74a] T.M. Mata, A.A. Martins, N.S. Caetano, Microalgae for biodiesel production and other applications: a review, Renew. Sustain. Energy Rev. 14 (1) (2010) 217-232.

[74b] A. Taleb, J. Pruvost, J. Legrand, H. Marec, B. Le Gouic, B. Mirabella, B. Legeret, S. Bouvet, G. Peltier, Y. LiBeisson, S. Taha, H. Takache, Development and validation of a screening procedure of microalgae for biodiesel production: application to the genus of marine microalgae Nannochloropsis, Bioresour. Technol. 177 (2015) 224-232.

[75] G. Van Vooren, F. Le Grand, J. Legrand, S. Cuine, G. Peltier, J. Pruvost, Investigation of fatty acids accumulation in Nannochloropsis oculata for biodiesel application, Bioresour. Technol. 124 (2012) 421-432.

[76] I.M. Remmers, A. Hidalgo-Ulloa, B.P. Brandt, W.A.C. Evers, R.H. Wijffels, P.P. Lamers, Continuous versus batch production of lipids in the microalgae Acutodesmus obliquus, Bioresour. Technol. 244 (Pt 2) (Nov 2017) 1384-1392.

[77] R. Kandilian, E. Lee, L. Pilon, Radiation and optical properties of Nannochloropsis oculata grown under different irradiances and spectra, Bioresour. Technol. 137 (2013) 63-73.

[78] J. Pruvost, J.F. Cornet, L. Pilon, Large-scale production of algal biomass: photobioreactors, in: F. Bux, Y. Chisti (Eds.), Algae Biotechnology: Products and Processes, Springer, 2016, pp. 41-66.

[79] J. Pruvost, F. Le Borgne, A. Artu, J.-F. Cornet, J. Legrand, Industrial photobioreactors and scale-up concepts, in: Advances in Chemical Engineering-Photobioreaction Engineering, Vol. 48, Elsevier, 2016, pp. 257-310.

[80] C. Posten, C. Walter, Microalgal Biotechnology: Potential and Production, Walter de Gruyter GmbH, 2012.

[81] E. Lee, J. Pruvost, X. He, R. Munipalli, L. Pilon, Design tool and guidelines for outdoor photobioreactors, Chem. Eng. Sci. 106 (2014) 18-29.

[82] L. Pilon, H. Berberoglu, R. Kandilian, Radiation transfer in photobiological carbon dioxide fixation and fuel production by microalgae, J. Quant. Spectrosc. Radiat. Transf. 112 (17) (2011) 2639-2660.

[83] J.U. Grobbelaar, Factors governing algal growth in photobioreactors: the "open" versus "closed" debate, J. Appl. Phycol. 21 (5) (2008) 489-492. 
[84] S. Hindersin, M. Leupold, M. Kerner, D. Hanelt, Key parameters for outdoor biomass production of Scenedesmus obliquus in solar tracked photobioreactors, J. Appl. Phycol. (2014).

[85] V. Goetz, F. Le Borgne, J. Pruvost, G. Plantard, J. Legrand, A generic temperature model for solar photobioreactors, Chem. Eng. J. 175 (2011) 443-449.

[86] Hindersin S. Photosynthetic efficiency of microalgae and optimization of biomass production in photobioreactors. 2013, PhD Thesis, Universität Hamburg.

[87] M.A. Borowitzka, Commercial production of microalgae: ponds, tanks, and fermenters, Prog. Ind. Microbiol. 35 (1999) 313-321.

[88] B. Perrier, C. Crampon, O. Guézet, et al., Production of a methyl ester from the microalgae Nannochloropsis grown in raceways on the French west coast, Fuel 153 (2015) 640-649.

[89] A. Muller-Feuga, M. Lemar, E. Vermel, R. Pradelles, L. Rimbaud, P. Valiorgue, Appraisal of a horizontal twophase flow photobioreactor for industrial production of delicate microalgae species, J. Appl. Phycol. 24 (3) (2012) 349-355.

[90] A. Taleb, J. Legrand, H. Takache, S. Taha, J. Pruvost, Investigation of lipid production by nitrogen-starved Parachlorella kessleri under continuous illumination and day/night cycles for biodiesel application, J. Appl. Phycol. 30 (2018) 761-772.

[91] M. Cuaresma, M. Janssen, E.J. Van den End, C. Vilchez, R.H. Wijffels, Luminostat operation: a tool to maximize microalgae photosynthetic efficiency in photobioreactors during the daily light cycle? Bioresour. Technol. 102 (17) (2011) 7817-7878.

[92] M. Cuaresma, M. Janssen, C. Vilchez, R.H. Wijffels, Horizontal or vertical photobioreactors? How to improve microalgae photosynthetic efficiency, Bioresour. Technol. 102 (8) (2011) 5129-5137.

[93] P.M. Slegers, M.B. Lösing, R.H. Wijffels, G. van Straten, A.J.B. van Boxtel, Scenario evaluation of open pond microalgae production, Algal Res. 2 (4) (2013) 358-368.

[94] P.M. Slegers, P.J.M. van Beveren, R.H. Wijffels, G. van Straten, A.J.B. van Boxtel, Scenario analysis of large scale algae production in tubular photobioreactors, Appl. Energy 105 (2013) 395-406.

[95] P.M. Slegers, R.H. Wijffels, G. Van Straten, A.J.B. Van Boxtel, Design scenarios for flat panel photobioreactors, Appl. Energy 88 (2011) 3342-3353.

[96] J.F. Cornet, C.G. Dussap, J.J. Leclercq, Simulation, design and model based predictive control of photobioreactors, in: M.H.a.P. Thonart (Ed.), Focus on Biotechnology. Engineering and Manufacturing for Biotechnology, Vol. 4, Kluwer Academic, Dordrecht, 2001, pp. 227-238.

[97] F.G. Acién Fernández, J.M. Sevilla, J.A. Sánchez Pérez, E. Molina Grima, Y. Chisti, Airlift-driven external-loop tubular photobioreactors for outdoor production of microalgae: assessment of design and performance, Chem. Eng. Sci. 56 (8) (2001) 2721-2732.

[98] W.J. Oswald, Large-scale algal culture systems (engineering aspects), in: M. Borowitska (Ed.), Microalgal Biotechnology, Cambridge University Press, Cambridge, 1988, pp. 357-394.

[99] E. Molina, J. Fernández, F.G. Acién, Y. Chisti, Tubular photobioreactor design for algal cultures, J. Biotechnol. 92 (2) (2001) 113-131.

[100] P. O, Photobioreactors: production systems for phototrophic microorganisms, Appl. Microbiol. Biotechnol. 57 (3) (2001) 287-293.

[101] G.C. Chini Zitelli, R. Pastorelli, M.R. Tredici, A modular flat panel Photobioreactor (MFPP) for indoor mass cultivation of Nannochloropsis sp. under artificial illumination, J. Appl. Phycol. 12 (3-5) (2000) 521-526.

[102] G. Chini Zittelli, L. Rodolfi, N. Biondi, M.R. Tredici, Productivity and photosynthetic efficiency of outdoor cultures of Tetraselmis suecica in annular columns, Aquaculture 261 (3) (2006) 932-943.

[103] J. Doucha, K. Livansky, Productivity, $\mathrm{CO}_{2} / \mathrm{O}_{2}$ exchange and hydraulics in outdoor open high density microalgal (Chlorella sp.) photobioreactorsoperated in a Middle and Southern European climate, J. Appl. Phycol. 18 (2006) 811-826.

[104] A. Richmond, Z. Cheng-Wu, Optimization of a flat plate glass reactor for mass production of Nannochloropsis sp. outdoors, J. Biotechnol. 85 (3) (2001) 259-269.

[105] S. Hindersin, M. Leupold, M. Kerner, D. Hanelt, Irradiance optimization of outdoor microalgal cultures using solar tracked photobioreactors, Bioprocess Biosyst. Eng. 36 (3) (2013) 345-355.

[106] C.H. Hsieh, W.T. Wu, A novel photobioreactor with transparent rectangular chambers for cultivation of microalgae, Biochem. Eng. J. 46 (3) (2009) 300-305.

[107] J.C. Ogbonna, H. Yada, H. Masui, H. Tanaka, A novel internally illuminated stirred tank photobioreactor for large-scale cultivation of photosynthetic cells, J. Ferment. Bioeng. 82 (1) (1996) 61-67. 
[108] J.W. Zijffers, M. Janssen, J. Tramper, R.H. Wijffels, Design process of an area-efficient photobioreactor, Mar. Biotechnol. 10 (2008) 404-415.

[109] E.W. Becker, Microalgae: Biotechnology and Microbiology, Cambridge, Cambridge University Press, 2008.

[110] W.J. Oswald, The coming industry of controlled photosynthesis, Am. J. Public Health Nations Health 52 (2) (1962) 235-242.

[111] Ben-Amotz A. Large-scale open algae ponds. NREL-AFOSR Joint Workshop on Algal Oil for Get Fuel Production 2008.

[112] H. Hadiyanto, S. Elmore, T. Van Gerven, A. Stankiewicz, Hydrodynamic evaluations in high rate algae pond (HRAP) design, Chem. Eng. J. 217 (2013) 231-239.

[113] K. Liffman, D.A. Paterson, P. Liovic, P. Bandopadhayay, Comparing the energy efficiency of different high rate algal raceway pond designs using computational fluid dynamics, Chem. Eng. Res. Des. 91 (2) (2013) 221-226.

[114] D. Chiaramonti, M. Prussi, D. Casini, et al., Review of energy balance in raceway ponds for microalgae cultivation: re-thinking a traditional system is possible, Appl. Energy 102 (2013) 101-111.

[115] R. Hreiz, B. Sialve, J. Morchain, R. Escudié, J.-P. Steyer, P. Guiraud, Experimental and numerical investigation of hydrodynamics in raceway reactors used for algaculture, Chem. Eng. J. 250 (2014) 230-239.

[116] T.J. Lundquist, I.C. Woertz, N.W.T. Quinn, J.R. Benemann, A Realistic Technology and Engineering Assessment of Algae Biofuel Production, Energy Biosciences Institute, Berkeley, CA, 2010.

[117] M.E. Grima, A.F.G. Fernandez, G.F. Camacho, Y. Chisti, Photobioreactors: light regime, mass transfer, and scaleup, J. Biotechnol. 70 (1999) 231-247.

[118] M. Morweiser, O. Kruse, B. Hankamer, C. Posten, Developments and perspectives of photobioreactors for biofuel production, Appl. Microbiol. Biotechnol. 87 (4) (2010) 1291-1301.

[119] Pruvost J, Le Borgne F, Artu A, Legrand J. Development and characterization of a thin-film solar photobioreactor (AlgoFilm (C) based on process intensification principles. Algal Res. 2017(21):120-137.

[120] A.C. Techniques, Photobioreactors and Fermentors: The Light and Dark Sides of Growing Algae, Academic Press, 2005. 
